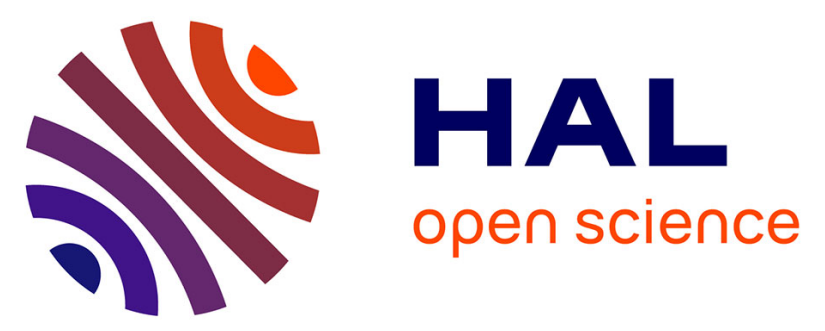

\title{
Quantitative prediction of discrete element models on complex loading paths
}

Luc Sibille, Pascal Villard, Félix Darve, Rodaina Aboul Hosn

\section{To cite this version:}

Luc Sibille, Pascal Villard, Félix Darve, Rodaina Aboul Hosn. Quantitative prediction of discrete element models on complex loading paths. International Journal for Numerical and Analytical Methods in Geomechanics, 2019, 43 (5), pp.858-887. 10.1002/nag.2911 . hal-02059431

\section{HAL Id: hal-02059431 https://hal.science/hal-02059431}

Submitted on 11 Oct 2019

HAL is a multi-disciplinary open access archive for the deposit and dissemination of scientific research documents, whether they are published or not. The documents may come from teaching and research institutions in France or abroad, or from public or private research centers.
L'archive ouverte pluridisciplinaire HAL, est destinée au dépôt et à la diffusion de documents scientifiques de niveau recherche, publiés ou non, émanant des établissements d'enseignement et de recherche français ou étrangers, des laboratoires publics ou privés. 


\title{
Quantitative prediction of discrete element models on complex
}

\section{loading paths}

\author{
Luc Sibille*1 | Pascal Villard $^{1}$ | Félix Darve ${ }^{1}$ | Rodaina Aboul Hosn ${ }^{2}$
}

${ }^{1}$ Univ. Grenoble Alpes, CNRS, Grenoble
INP, 3SR, F-38000 Grenoble, France
${ }^{2}$ IRSTEA, Research Unit Recover, 13182
Aix-en-Provence cedex 5, France

Correspondence

*Corresponding author, Email:

luc.sibille@3sr-grenoble.fr

\begin{abstract}
Summary
The ability of discrete element models to describe quantitatively (and not only qualitatively) the constitutive behaviour of a dense sand is assessed in this paper. Two kinds of 3D discrete models are considered. Both consider spheres as elementary particles. Nevertheless the first model implements a contact law with rolling resistance whereas the second takes into account clumps made of two spheres. The discrete models are calibrated and validated from mechanical tests performed on a dense Hostun sand with a true triaxial apparatus. The calibration is carried out from axi-symmetric drained compression tests, while the validation is discussed from monotonic and cyclic stress proportional loading paths and from a circular stress path in the deviatoric stress plane. The quality of the predictions of the discrete models are evaluated by comparison with the predictions given with advanced phenomenological constitutive relations, mainly an incrementally non-linear relation. Predictions given by the discrete models are remarkable, particularly when it is put in perspective with respect to the very few number of mechanical tests required for their calibration. However, these results and conclusions were reached in enabling conditions and some limitations of such discrete models should be kept in mind.
\end{abstract}

\section{1 | INTRODUCTION}

\section{1 | From continuous to discrete constitutive modeling}

Granular media are a typical example of media with a discernable discrete microstructure, constituted by the grain assembly.

For some macroscopic aspects of their behavior the existence of this microstructure can not be ignored, like for instance the thickness of shear bands (which depends drastically on grain size among other less important parameters) or phenomena as segregation, erosion, etc... The most manageable way to take into account this microstructure while preserving the framework of 
continuous mechanics is certainly to enrich the classical description by introducing an internal length through second gradient theories ${ }^{\frac{1}{1}}$, Cosserat mechanics ${ }^{\sqrt{2}}$ or non-local integral constitutive relations ${ }^{3}$. However some difficulties remain still present like the questions of bifurcations, instabilities, losses of uniqueness and failure modes, technically intricate to be efficiently solved in the framework of enriched continuum mechanics. Another type of difficulties concern the question of the manageable description of the behavior of granular materials by phenomenological elasto-plastic constitutive relations. This behavior is essentially incrementally non-linear (the relation between the incremental stress and the incremental strain is non-linear) ${ }^{4}$, what induces some difficulties in the mathematical - and thus also the numerical - treatment of this kind of relations. Let us recall that, in elasto-plasticity, the rheological functional is non-differentiable ${ }^{5}$. Besides, beyond the incremental non-linearity, the question of the definition and evolution laws of the plastic internal variables is very intricate particularly in the case of non-proportional or cyclic loading paths.

Facing these irreducible difficulties to describe the elasto-plastic behavior of granular media in a continuum mechanics framework, another way appears as promising by considering molecular dynamics method or the so-called direct numerical simulations (DNS). In the case of granular materials, the application of molecular dynamics technics to grain assemblies has led to a class of numerical methods called by the generic name of "Discrete Element Methods" (DEM). Molecular dynamics is essentially based on the idea that the complexity of the real world is due to the extremely big number of atoms, molecules, grains, ... in interaction, and not to the complexity of the interaction laws themselves. This is particularly well verified for granular media, where a simple dry Coulombian intergranular friction (one single mechanical parameter !) allows to describe the whole complexity of the mechanical behavior of an assembly of millions of grains in contact 6 (617. However a question is rising here: in which extent are DEM models able to describe quantitatively (thus, not only qualitatively) this complex behaviour as it is observed experimentally for non-proportional loading paths or in cyclic loading ?

DEM has been widely used in geomechanics as a tool to perform numerical experiments on a representative elementary volume (REV) in order to investigate, on one hand, the constitutive behaviour of geomaterials at the scale of the REV 890 and, on the other hand, the underlying mechanisms at the grain scale or the contact scale ${ }^{710}$. Besides, the modelling or large scale in situ problems for engineering applications relies usually on a continuum approach with phenomenological constitutive relations embedded in finite element or finite difference methods. Today, such continuum approaches require only a relatively low computational cost. Nevertheless, the permanently growth of the computing power, the optimisation of the DEM code $\frac{11}{11}$, and the optimisation of the numerical methods themselves (by using for instance a particle refinement method ${ }^{12}$ or a multi-domain FEM-DEM method ${ }^{13}$ ) offer today the possibility to tackle with the DEM boundary value problems defined in the laboratory (as for instance a cone penetration test in a calibration chamber ${ }^{\sqrt{14}}$ ) or in situ (such as soil reinforcement with geosynthetics ${ }^{15}$ or rigid 
inclusions $\frac{16}{16}$ for example). In a near future the modelling or large scale field applications should even be feasible with numerical methods involving, at least partially, some discrete elements. In this context, the question of the ability of DEM models to describe quantitatively the behaviour of geomaterials seems particularly relevant.

\section{2 | A benchmark inspired from the Cleveland workshop}

To assess properly the predictive ability of constitutive models, the most convincing way is certainly to apply the methodology which was taken into account in the eighties to compare each other the predictive capacities of the available constitutive relations at that time. The most important step in this methodology was probably the organization of international workshops specifically devoted to this comparison. The last one was organized in Cleveland in Case Western Reserve University (CWRU) on 22-24 july $1987^{17}$. 27 different phenomenological elasto-plastic constitutive relations have been compared as objectively as possible. For that, some calibration tests were sent, before the workshop, to the 27 authors or users of constitutive relations for sands to allow them to calibrate their models by determining the constitutive parameters. In the same time, some predictions on other loading paths were asked and they should be sent to CWRU with a deadline fixed before the workshop. Then, during the workshop, the predictions produced by the 27 constitutive relations have been compared to the experimental results, just revealed at the workshop time. The patronage of the workshop was assured by the American NSF, ASCE, ASTM and French CNRS. What will interest us in the present paper are the prediction paths, for which DEM predictions are here presented and analysed.

Three kinds of prediction paths have been considered for Cleveland workshop:

(i) Proportional stress paths in plane stress conditions with a constant Lode's coefficient (usually called "b") equal to 0.3 and 0.7 (paths applied in a true triaxial machine),

(iia) A cyclic torsional path with 5 cycles, the last one being pursued until failure (paths applied in a hollow cylinder apparatus),

(iii) A 3D stress path, circular in a deviatoric stress plane and followed twice (paths applied in the true triaxial apparatus).

The main conclusions have been the following ones roughly speaking:

(i) For the proportional stress paths, about $70 \%$ of the elasto-plastic constitutive relations gave satisfying results, more or less independently to the details of the models,

(iia) For the cyclic torsional path, not any model was able to produce reasonable quantitative predictions: the question of principal stress and strain axis rotation was a fully open question, 
(iii) For the 3D circular stress path, only incrementally non-linear models gave quantitatively acceptable results: for a strongly non-proportional path the incremental non-linearity of sand behavior plays a basic role.

To check the predictive capacities of DEM to model complex granular behavior, it is proposed in this paper to simulate by DEM the three above paths. In fact, paths (iia) have been replaced by other cyclic paths (iib) obtained in the same true triaxial apparatus as paths (i) and (iii), in order to be perfectly consistent for the calibration tests and the validation tests, therefore all performed on the same true triaxial machine and simulated by the same cubical numerical sample. Paths (iib) consist in proportional stress paths at constant mean pressure and involve from six to height loading/unloading cycles. Because quantitative predictions are sought here, it was necessary to consider DEM models able to be calibrated on lab tests performed on real sands. Two classical solutions are available to reproduce realistically the high friction angles of real sands: spherical grains with a rolling resistance or clump elements. Thus both these DEM models are considered in this paper.

Eventually the DEM results are plotted as well as the predictions produced by an incrementally non-linear phenomenological model, exactly as presented during Cleveland workshop. The comparison with more conventional elasto-plastic models is also considered in the case of the path (iii). The objective here is to give some insights into the following question: are the predictive capacities of DEM models (with very few mechanical parameters) better than the ones of an incrementally non-linear model (with many parameters), which has been considered as successful in Cleveland workshop ?

The outline of the paper follows logically. The considered sand and the true triaxial machine are briefly presented in section 2 Then both the DEM models and the phenomenological relations are also briefly recalled with their calibration procedures (sect. 3 and 4). The three validation paths (i), (iib) and (iii) are defined and the predictions, carried out by the DEM models and the phenomenological relation, are compared with the experimental results (sect. 5). Finally, main conclusions are discussed in section 6 .

\section{2 | EXPERIMENTAL CHARACTERIZATION OF HOSTUN SAND “RF”}

\section{1 | Hostun sand "RF"}

Two different sands were used for the Cleveland Workshop: the Reid Bedford sand and the Hostun sand "RF". Only the experimental results obtained with the Hostun sand are considered in this paper. A description of this sand is given by Flavigny $e t$ $a l .18$ and the main characteristics are recalled below.

The Hostun sand "RF" is composed of angular grains with a uniform grain size distribution (Fig. 1] and a mean grain size $D_{50}=0.35 \mathrm{~mm}$. Depending on the technique used the minimum void ratio was found in the range $e_{\min } \in[0.624 ; 0.648]$ (by excluding the moist tamping technique) and the maximum void ratio in the range $e_{\max } \in[0.961 ; 1.041]$. All the experimental 
tests used in this paper were performed from sand samples with an initial void ratio of 0.626 (or 0.636 , depending on the reference: Lanier and Zitouni ${ }^{10}$ or Zitouni ${ }^{20}$ respectively) corresponding to a very dense initial state.

\section{2 | The true triaxial apparatus}

Experimental tests were carried out with the true triaxial apparatus (TTA) ${ }^{20 \mid 19}$ developed at the former "Institut de Mécanique de Grenoble" presently called 3SR Laboratory. The TTA consists in a parallelepipedic box made of six rigid steel platens. Platens can move in such a way that the inside box deforms by staying parallelepipedic as shown in Figure 2 The sand sample, initially cubical $\left(10 \times 10 \times 10 \mathrm{~cm}^{3}\right)$ and enclosed inside a rubber membrane (with a cubical shape as well), is placed inside the box formed by the rigid platens. The interface between the outside face of the rubber membrane and the platens is lubricated to avoid shear stress development on the sample boundary. Consequently, only compressive normal stresses are applied by the platens on the six sample faces and stress and strain principal directions coincide with the normals to the platens. The translation of the platens in the direction normal to the platen/sample interface is performed with six electric motors synchronized two by two. Then, the three principal components of stresses or strains are controlled independently by controlling the kinematic of the platens: either directly for strains by adjusting the platen displacements, or indirectly for stresses thanks to a closed-loop control. Consequently, all loading programmes defined with principal values of stresses and/or strains can be applied.

The principle of the TTA is very close to the discrete numerical models used in this study and presented in the next section. In particular, in both cases the sample (or the elementary periodic cell) is parallelepipedic and each principal stress or strain component is independently controlled (or independently measured / computed). Moreover, for conventional axisymmetric triaxial apparatuses the initial cylindrical sample may take a barrel shape during the compression. Therefore, the description of the rubber membrane in the numerical model is required to take into account such a sample deformation. Concerning the TTA, as the sample keep at any times the shape of a parallelepiped there is no need to take into account a membrane effect

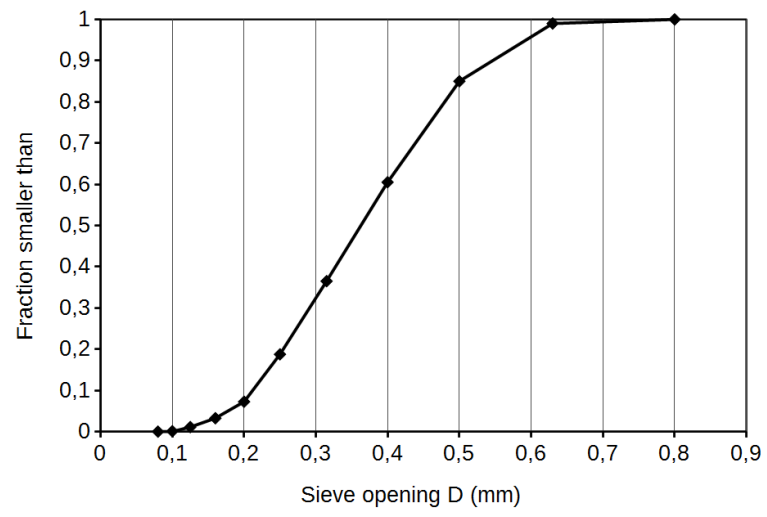

FIGURE 1 Grading curve of the Hostun sand "RF", after Flavigny et al.18. 
and the numerical modeling is rather simplified. For these different reasons the possible discrepancies between experimental soil responses and simulated ones due to differences in the experimental and numerical boundary conditions are, indeed not completely eliminated, but at least limited.

\section{3 | PREDICTIVE MODELS}

\section{1 | Phenomenological constitutive relation}

From a general point of view, phenomenological relations are built inside a mathematical framework given by continuum mechanics principles and laws, and by some assumptions issued from experiments usually performed on material samples. So the principle of determinism implies the existence of a tensorial relation between the stress increment tensor, $d \boldsymbol{\sigma}$, the strain increment tensor, $d \boldsymbol{\varepsilon}$, and the time increment, $d t$. Moreover, if only elasto-plastic materials are considered (rate-indepent media), this function is independent of $d t$ and homogeneous of degree 1. Now the application of Euler's Identity for homogeneous functions implies the existence of the elasto-plastic tangent tensors $\mathbf{M}$ or equivalently $\mathbf{N}^{21}$ :

$$
d \boldsymbol{\varepsilon}=\mathbf{M}(\mathbf{u}) d \boldsymbol{\sigma} \quad \text { or } \quad d \boldsymbol{\sigma}=\mathbf{N}(\mathbf{v}) d \boldsymbol{\varepsilon}
$$

where $\mathbf{u}=d \boldsymbol{\sigma} /\|d \boldsymbol{\sigma}\|$ and $\mathbf{v}=d \boldsymbol{\varepsilon} /\|d \boldsymbol{\varepsilon}\|$ represent the unit vectors in the directions respectively of the incremental stress and the incremental strain.

In the following a vectorial notation considering the six-dimensional related spaces is adopted for convenience. Then for example the strain vector (previously tensor) writes:

$$
d \varepsilon_{\alpha}=\left(d \varepsilon_{11}, d \varepsilon_{22}, d \varepsilon_{33}, \sqrt{2} d \varepsilon_{23}, \sqrt{2} d \varepsilon_{31}, \sqrt{2} d \varepsilon_{12}\right)
$$

Now by keeping only both the first terms in a Taylor's expansion of $\mathbf{M}$, we obtain the incrementally non-linear relation of second $\operatorname{order}^{22}$ :

$$
d \varepsilon_{\alpha}=M_{\alpha \beta}^{1} d \sigma_{\beta}+\frac{1}{\|d \boldsymbol{\sigma}\|} M_{\alpha \beta \gamma}^{2} d \sigma_{\beta} d \sigma_{\gamma}
$$
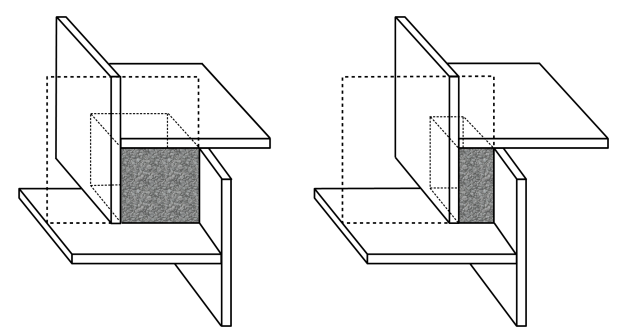

FIGURE 2 Principle of the true triaxial apparatus (TTA) with rigid platens, initial geometry on the left and deformed geometry on the right (after Lanier and Zitouni ${ }^{19}$ ). 
In equation (3), it is easy to check the incremental non-linearity of the relation (describing the irreversibility of plastic stains) and its homogeneity of degree 1 (describing the non-viscous character of the elasto-plastic strains) with respect to six variables $d \sigma_{\beta}$.

With some additional assumptions (like the orthotropy of the incremental relation), both the tensors $\mathbf{M}^{1}$ and $\mathbf{M}^{2}$ can be calibrated on triaxial tests in compression and in extension. By taking into account all types of constitutive parameters, sixteen coefficients have to be determined in the fully general calibration procedure.

This model has been utilised by Darve and Dendani ${ }^{23}$ in Cleveland workshop. The predictions of this model called "INL2", as presented in the workshop, will be recalled in this paper for quantitative comparisons with two DEM models as discussed in the introduction and presented in the next section.

Another way to build an elasto-plastic relation consists to decompose additively the incremental strain into an elastic part and a plastic part. Then, in a first step, an elastic tensor is introduced to express the elastic incremental strain as a function of the incremental stress. An elastic stress domain has to be defined, where the elastic model will be applied. From the boundary of this domain (the so-called "elastic limit" or equivalently "loading surface") some plastic strains appear. The direction of these strains is given by a flow rule (generally considered as non-associated for geomaterials), expressed from the normal to a plastic potential. Eventually the consistency equation allows to fully determine the incremental plastic strain by giving its norm. But, even outside the elastic domain, the experiments show that, for an unloading, the strains are essentially elastic: this can be viewed also as the consequence of the incremental non-linearity of elasto-plasticity. Thus a loading-unloading criterion has to be introduced which is related to the normal direction to the boundary of the elastic domain. Eventually the hardening variables allow to describe the crucial evolution of this boundary (and of the plastic potential) with the loading path and/or the stress state. The hardening is called "isotropic" if the elastic limit is growing homothetically with the stress state and "kinematical" if the elastic limit is translating. Both these kinds of elasto-plastic relations are also considered in this paper, in the case of the circular loading path in the deviatoric stress plane (Section 5.3 by presenting the predictions in Cleveland Workshop of elasto-plastic relations with an isotropic hardening (model called "EPIH") and a kinematical hardening in addition to the isotropic one (called "EPKH").

\section{2 | Discrete numerical models}

Two 3D discrete numerical models have been used, they are based on the discrete element method (DEM) as introduced by Cundall and Strack ${ }^{24}$. A classical iterative process that successively alternates the resolution of Newton's second law of motion for each grain (or particle) and the actualization of the interaction forces at each inter-granular contact point is used to achieve the numerical simulation. The elementary particles implemented in both models are spherical particles. However, soil grain shape (angularity, anisotropy) plays a major role in the constitutive behaviour of soils. In particular realistic shear strengths of 
soils cannot be described with a straightforward implementation of spherical particles in the DEM, resulting in an excessive rotation of particles.

The two models considered here differs mainly in the way to overcome this issue. In the first model, DEM-R, a resistance to the relative rolling between two particles in contact is added to the contact law. This additional constraint to the contact law is an indirect way to reflect the influence of real angular grains on their motion. In the second model, DEM-C, the non spherical shape of the grains is more directly taken into account by forming rigid clusters of elementary spherical particles grouped two by two for the sake of simplicity.

Of course it is possible today to implement in the DEM more advanced geometrical models of discrete particles mimicking quite closely the actual grain shape. However such advanced geometrical models of particles lead to a strong increase of the computational cost (already not negligible for a classical DEM model) and the implicit introduction of additional geometric parameters in the model. The choice have been made here to focus on relatively light DEM models involving each one only four or five mechanical parameters to emulate cohesionless granular materials and which are currently used to study boundary value

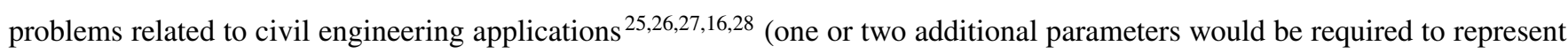
cohesive materials).

\subsection{1 | Initial porosity of the discrete models}

The initial porosity is an important state parameter in soil mechanics since it reflects how a sheared soil sample is prone to producing dilatation and higher peak shear strength, depending on the difference between the initial density and the density at the critical state. In the same way, the initial porosity of the numerical granular assemblies will play a fundamental role and its choice is not necessarily straightforward. Therefore, the ways to choose the initial porosity for the DEM-R and DEM-C models are shortly discussed here after. Three main different options can be followed.

First, the initial absolute porosity of the numerical sample could be assigned as identical to the initial porosity of the real soil sample. The hypothesis behind this option is that the numerical assembly should produce the same volumetric deformation (porosity change) as the soil sample for an initial numerical porosity identical to the real one. If numerical and real grain shapes are different (e.g. spheres instead of angular sand grains) there is no reason to reach in these conditions the same volumetric deformation (unless the volumetric deformation is corrected with some ad hoc parameters of the model). If the grain shape is very different we could even imagine to obtain opposite volumetric deformations (contraction instead of dilation for instance).

Second, the relative density of the numerical sample (computed with respect to some numerical maximum and minimum initial density states) could be fixed to match the relative density of the real soil sample. Similarly, it would assume that an identical relative density leads to an identical volumetric deformation. The latter implies that maximum and minimum initial density states of the numerical assembly emulate the same volumetric deformations as the ones measured from soil samples 
initially at the maximum and minimum states of density respectively. Again this is not insured if the shape of the grains (and the complete micro-structure) is not reproduced numerically. Nevertheless, the application of this option shows that generally volumetric deformations simulated with the model in these conditions is relatively close to the real ones.

A third option consists in defining the initial absolute numerical porosity as the one resulting in the same volumetric deformation (porosity change) as the real one, and depending on the difference between the initial porosity and the one at the critical state (depending itself on the particle shapes). In this context, it is considered that the porosity does not have an absolute meaning but is defined relatively to the dilative properties of the medium.

Two slightly different strategies have been followed for the two DEM models. Concerning the DEM-C model the initial porosity is chosen with a process half-way between the options two and three. At the beginning, the initial relative density of the model is fixed equal to the initial relative density of the soil sample. Then the initial density is adjusted from this starting point to approach more closely, if necessary, the volume change during the compression. For the DEM-R model the initial porosity is chosen according to the third option. These strategies for the choice of the porosity are embedded in the methodologies of calibration of the models presented in the two next sections.

\subsubsection{DEM with rolling resistance (DEM-R)}

For the discrete model, identified as DEM-R in this paper, an inter-granular contact law involving a rolling moment acting against the relative rotation of the particles in contact is considered in addition to the more classical Coulombian friction model.

For a couple of interacting particles presenting an overlap $\delta_{n}$ and a normal $\vec{n}$ to the tangent contact plane the normal and tangential contact forces write respectively (see Figure 3 a):

$$
\vec{F}_{n}=k_{n} \delta_{n} \vec{n}
$$

$$
\Delta \vec{F}_{t}=-k_{t} \Delta \vec{u}_{t}
$$

where $k_{n}$ and $k_{t}$ are normal and tangential stiffnesses considered constant. $\vec{F}_{t}$ has to be computed incrementally from the relative tangential displacement $\vec{u}_{t}$ at the contact point due to the non linearity introduced by the Coulombian friction. The latter implies that:

$$
\left\|\vec{F}_{t}\right\| \leq\left\|\vec{F}_{n}\right\| \tan \varphi_{c}
$$

with $\varphi_{c}$ the contact friction angle. 


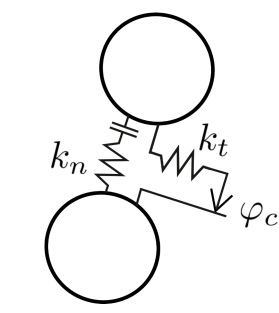

(a) (b)

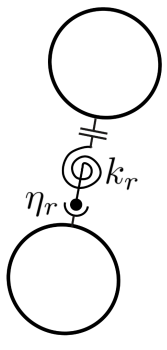

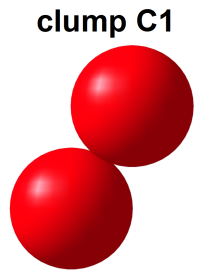

(c)

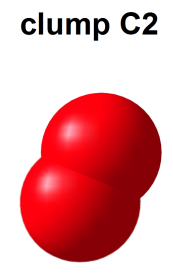

(d)

FIGURE 3 Rheological models of the contact laws and clumps of particles: (a) model of the contact in the normal and tangential direction; (b) model of the rolling resistance at contact; (c) \& (d) clump C1 \& C2 with a distance between particle centers equal to $D$ or $D / 2$ respectively (where $D$ is the particle diameter).

The rolling moment at contact, $\vec{M}_{r}$, is defined from a rolling stiffness $k_{r}$ (also considered as constant) and acts against the relative rolling rotation of the particles $\vec{\theta}_{r}$ (Figure 3 ):

$$
\Delta \vec{M}_{r}=-k_{r} \Delta \vec{\theta}_{r}
$$

It is limited by a plastic threshold expressed as:

$$
\left\|\vec{M}_{r}\right\| \leq\left\|\vec{F}_{n}\right\| \eta_{r} \min \left(R_{1}, R_{2}\right)
$$

where $R_{1}$ and $R_{2}$ are the radii of the two spheres in contact and $\eta_{r}$ is the plastic rolling coefficient.

Finally, to make the macroscopic elastic parameters of the granular assembly independent of the particle size, the contacts stiffnesses are defined from a stiffness modulus $E_{c}$ and dimensionless tangential and rolling coefficients, $\alpha_{t}$ and $\alpha_{r}$ respectively:

$$
k_{n}=2 E_{c} \frac{R_{1} R_{2}}{R_{1}+R_{2}} ; \quad k_{t}=\alpha_{t} k_{n} ; \quad k_{r}=\alpha_{r} R_{1} R_{2} k_{t}
$$

Consequently the five mechanical parameters of the model DEM-R are: $\eta_{r}, \varphi_{c}, E_{c}, \alpha_{t}$ and $\alpha_{r}$.

If the elastic parameters are chosen sufficiently close to the rigid limit then the plastic properties of the granular assembly at the macroscopic scale depend on the contact plastic parameters $\left(\eta_{r}\right.$ and $\left.\varphi_{c}\right)$ only and the macroscopic elastic properties are fixed by the elastic contact properties $\left(E_{c}, \alpha_{t}\right)$. The choice of the mechanical parameters has been performed in accordance with this

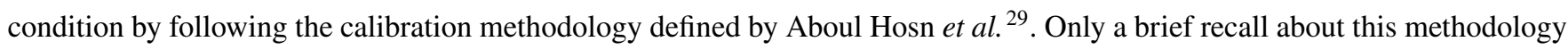
is given here.

- The first step consists in calibrating $\eta_{r}$ to fit the shear strength at large deformation (at critical state) which depends only on $\eta_{r}$ (if $\varphi_{c}$ is not too low ${ }^{29}$ ). In this objective, a cloud of non-overlapping particles is isotropically compacted until reaching the target confining pressure. $\varphi_{c}$ is fixed arbitrarily to $30^{\circ}$ in order to start from a medium dense material (but in all cases 
shear strength at large deformation is independent of the initial density) and the value of $\eta_{r}$ is calibrated to fit the critical shear strength.

- In a second step, the volumetric deformation (dilatancy/contractancy) and the peak shear strength are approached as close as possible by tuning the initial sample density. In the present case, where a dense sand sample is considered, the initial density is progressively increased by lubricating the contact during the compaction phase. The latter is done by choosing a contact friction for compaction lower than the nominal contact friction angle used to simulate the mechanical test. Further information concerning the initial compaction of numerical granular assemblies via an isotropic compaction can be found for instance in the works of Combe and Roux ${ }^{30}$ or Aboul Hosn et al. 29 .

- In a third step the nominal value of the contact friction angle is calibrated (if necessary) to improve the reproduction of the volumetric deformation and the peak shear strength.

- Finally in a fourth step the contact stiffness modulus $E_{c}$ and the tangential coefficient $\alpha_{t}$ are adjusted to improve, if necessary, the reproduction of the slopes at low deformation of the macroscopic stress-strain response and of the volume variation respectively (see for instance the plots of $\sigma_{1} / \sigma_{3}$ vs $\varepsilon_{1}$ and $\Delta V / V$ vs $\varepsilon_{1}$ in Figure 5 , reflecting partly the elastic properties of the soil sample.

It is worth nothing that no calibration is performed on the rolling coefficient $\alpha_{r}$ as it does not influence the macroscopic properties if it is sufficiently high (i.e. close enough to the rigid limit). This can be checked simply by repeating simulations with higher values of $\alpha_{r}$, the macroscopic mechanical response should be unchanged. Besides, this calibration methodology emphasized the importance of:

- the plastic rolling coefficient, $\eta_{r}$, which constitutes an indirect way to describe the angular grain shapes; the latter is known to be a preponderant factor in the load bearing capacity of a granular soil, as a sand for instance

- the initial density of the sample, which is classically known to be a determining state parameter to describe the mechanical response of granular matters.

\subsection{3 | DEM with clump (DEM-C)}

For the discrete model identified in this paper as DEM-C, except the fact that clumps of two spheres were used to avoid rolling, contact laws and numerical calibration process are rather similar than those previously described. By sake of simplicity and in order to approach reasonably the mechanical macroscopic behaviour of the experimental material, we opted, for this study, for numerical samples composed of unbreakable clumps of two juxtaposed (Clump C1) or overlapped (Clump C2) particles of 
same diameter $D$ (Figures $3 \& \&$ thespectively). For Clumps $C 2$ the distances between the centers of the two spheres of one cluster are equal to $D / 2$.

The contact interaction law between clumps is actually defined between the contacting spheres belonging respectively to each clump. Normal and tangential contact forces between spheres (Figure 3 a) are computed according to equations 4 and 5 and by taking into account the Coulombian friction as expressed in equation 6 There is no rolling resistance at contact point between two spheres considered for the DEM-C model. Then, the DEM-C model includes 4 mechanical parameters $\varphi_{c}, E_{c}, \alpha_{t}$ (defined in Equation 9) and the distance between sphere centres composing the clumps.

The creation of numerical samples consists in positioning the clusters randomly in a defined volume at a fixed density using the process of radius expansion with a decrease of friction (REDF process). This process is started by an initial randomly positioning of small clumps in a loose state. The radius of the particles composing the clumps is progressively increased and leads to the rearrangement of the granular assembly. The use of a particular value of the friction during the radius expansion process leads to a particular value of the porosity (detail descriptions of the REDF process were given by Chareyre and co-workers $\frac{3132}{32}$ ).

The calibration process for DEM-C ${ }^{33}$, based on the comparison between numerical and experimental triaxial tests, follows a rather similar procedure as the one given for DEM-R.

- Firstly, the main parameter dealing with the rolling resistance mechanisms is adjusted, i.e. the elongation (distance between particle centres) of the clumps, in order to reach the experimental shear strength at large deformations.

- Generally, values of the numerical relative density identical to the experimental ones give numerical answers, in terms of volume variation during shearing, close to the real one. Hence, in a second step, the initial relative porosity, assigned at the beginning as equal to the relative porosity of the experiment, is now tuned from this starting point to approach as close as possible the experimental volume change during the compression.

- In a third step the nominal value of the contact friction angle (i.e. the one used to simulate the triaxial compression) is defined in order to obtain the right value of the peak shear strength.

- Finally the contact stiffness modulus $E_{c}$ and the tangential coefficient $\alpha_{t}$ are fixed to approach the experimental elastic characteristics of the soil sample. 


\section{4 | MODEL CALIBRATIONS ON AXI-SYMMETRIC COMPRESSION AND EXTENSION}

\section{TESTS}

\section{1 | Experimental data for calibrations}

Axisymmetric and drained (i.e. $\sigma_{2}=\sigma_{3}=p_{0}=c s t$.) compression and extension test results were provided for the calibration of the models for the benchmark of Cleveland. Three different confining pressures were considered $\left(p_{0}=200 ; 350 ; 500 \mathrm{kPa}\right)$. Compression tests were performed for all the confinements, whereas extension tests were carried out for confinements of $200 \mathrm{kPa}$ and $500 \mathrm{kPa}$ only. Characteristics of calibration tests are resumed in Table 1 For some tests (CH1 and CH5) unloading-reloading cycles were performed.

Note that repeatability tests were presented by Zitouni ${ }^{20}$ but they are not reproduced in this paper to avoid an excessive number of figures. The test results retained for the calibration were, among others, the ones where the strain localization was the most delayed in order to provide experimental data the most representative of the actual constitutive behaviour of the Hostun sand.

An isotropic compression (named $\mathrm{CH} 6^{1734}$ ) including several unloading-reloading cycles was also given as a calibration test for the Cleveland benchmark. However, none of the calibration procedures for the models considered in this paper (INL2 and DEM models) requires an isotropic compression path. Consequently, the experimental data from the isotropic compression have not been used here.

\section{2 | Calibration of the phenomenological model INL2}

The whole methodology of calibration of the model INL2 is not given here for a sake a concision. A complete presentation of the calibration would require a detailed presentation of the model and its internal constituents which constitutes actually an article in itself. The description of the calibration on the Hostun sand RF has been detailed by Darve and Dendani ${ }^{23}$. We recall here that the calibration of the model INL2 requires three axisymmetric drained compressions and three axisymmetric drained extensions

TABLE 1 Characteristics of the axi-symmetric drained compression and extension tests; the names correspond to the names of the experimental tests 17734 .

\begin{tabular}{lcc}
\hline $\begin{array}{l}\text { Name } \\
\text { (Exp. test) }\end{array}$ & Type & $\begin{array}{c}\text { Confinement } \\
p_{0}(\mathbf{k P a})\end{array}$ \\
\hline $\mathrm{CH} 1$ & Compression & 200 \\
$\mathrm{CH} 2$ & Compression & 500 \\
$\mathrm{CH} 3$ & Extension & 200 \\
$\mathrm{CH} 4$ & Extension & 500 \\
$\mathrm{CH} 5$ & Compression & 350 \\
\hline
\end{tabular}


performed respectively at three different confining pressures. As only two extension tests were provided, unloading/reloading cycles performed in test $\mathrm{CH} 5$ at the intermediate confining pressure or $350 \mathrm{kPa}$ were also used here for the calibration of the model.

The results of the calibration of the model is displayed in Figure 4 In this figure, as the other ones of this paper, experimental results are displayed with thick magenta (or grey in black and white printings) lines; whereas simulated responses are displayed with thin, generally black, lines. The calibration of the INL2 relation leads to the identification of sixteen constitutive parameters of the model (also given in Darve and Dendani ${ }^{23}$ ).

The simulated responses to the different compression and extension tests fit particularly well the experimental data. For large deformations (typically for $\varepsilon_{1}>10 \%$ ) the simulated shear strength starts to deviates from the experimental one. For this range of deformation the localization of the deformations is generally well developed ${ }^{20}$ accentuating the softening behaviour in the experimental stress-strain response. Localization of deformation is not taken into account by such a constitutive modelling aiming to describe the intrinsic mechanical behaviour of the soil. The description of the localization (and the related stressstrain softening) would require to consider a boundary value problem with a numerical method (as the finite element or the finite difference method) integrating the INL2 model (or other phenomenological constitutive relations).

\section{3 | Calibration of the discrete model DEM-R}

A parallelepipedic periodic cell including 10,000 spherical particles have been considered for this study (Salot et al. ${ }^{33}$ shown that a minimum number of 8,000 particles should be considered in 3D to constitute a representative elementary volume). The particle size distribution of the numerical sample is almost identical to that of the Hostun sand RF displayed in Figure 1 Only the extreme parts of the distribution (i.e. particles smaller than $0.125 \mathrm{~mm}$ and larger than $0.63 \mathrm{~mm}$ ), representing a very low mass fraction of the soil, have been discarded. The calibration of the model as described in section 3.2.2 requires a single compression test. Consequently, even if several experimental compressions (and extensions) are available, the compression test performed at the intermediate confining pressure of $350 \mathrm{kPa}$ (test CH5) was the only one used for the calibration. The identified model parameters are given in Table 2 for an initial state of the numerical granular assembly at a void ratio $e=0.563$.

The simulated responses to all the triaxial compression and extension loadings are presented in Figure 5 Actually, the simulation of tests other than test $\mathrm{CH} 5$, with the parameters identified from test $\mathrm{CH} 5$, can be seen here as a first set of validation of the model DEM-R. In the same way unloading/reloading cycles present in tests CH5 and CH1 were not used for calibration, as no parameters of the model are related to unloading/reloading cycles. Hence, their simulations constitute also real predictions. 
Model: INL2
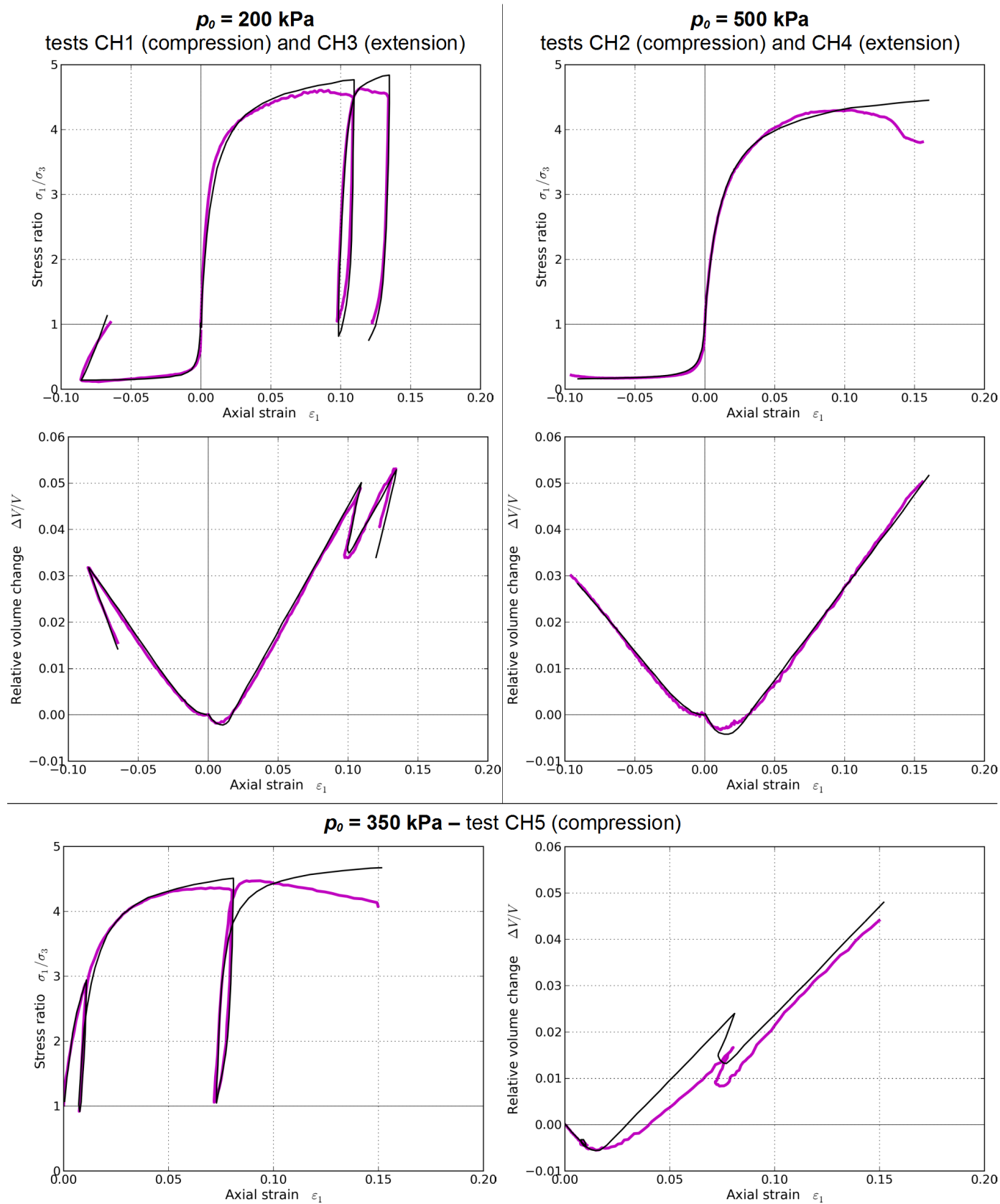

FIGURE 4 Calibration of the model INL2 from drained compression and extension tests. Experimental results are displayed with thick magenta (or grey in black and white printings) lines, whereas simulated responses are displayed with thin black lines. This plotting convention holds, generally, for all the figures in this paper.

General comments can be done for all the compressive tests (i.e. whatever the confining pressure). The model is slightly too stiff in the hardening regime. However, as no parameter of the model is directly related to this behaviour it is almost pointless to try to correct totally this difference with the experimental data. Besides, the volumetric response of the model is slightly less dilatant than the sand sample. The dilatancy depends, in the model, on the contact friction angle and on the initial density state. Nevertheless, contact friction angle and initial density impact also the axial stress peak and the chosen parameters result 
from the best compromise found between the description of the peak shear strength and the volumetric response. The shear strength of the test used for calibration (CH5) is well described whereas it is very slightly overestimated for the test $\mathrm{CH} 2$, where the confining pressure is higher, and more largely underestimated for the test $\mathrm{CH} 1$, performed at a lower confining pressure. Consequently, the model fails to describe accurately the change of mobilized friction angle with mean pressure. As discussed in Aboul Hosn et al. ${ }^{29}$ it may be related to the Coulombian friction model (Eq. 6) where the contact friction angle is independent of the normal contact force, which is of course consistent with the Coulombian model but may not be totally true (more likely for high confining pressure and easily degradable grains).

Concerning the unloading/reloading cycles, they are quite well described for test $\mathrm{CH} 1$ and the simulated response is slightly too soft (for both unloading and reloading paths) for the test CH5. However, here again no parameter is related to this cyclic response and the response is satisfactory knowing that no fitting is possible. In the same way, the simulated responses to extension tests $(\mathrm{CH} 3$ and $\mathrm{CH} 4)$ are pure predictions of the model with a good agreement with the experimental data.

\subsection{Calibration of the discrete model DEM-C}

The definition and the calibration of the models DEM-C and DEM-R have been performed independently by two different operators, limiting therefore possible interferences on the choice of the constitutive parameters for each model respectively.

For the DEM-C model a parallelepipedic assembly of 8,000 clumps, enclosed within six rigid and frictionless walls, has been used. The grading of the numerical material is defined by a uniform distribution in weight of the clump sizes (with a size ratio between the large and small clumps of 2), minimizing therefore the number of small particles and the duration of the numerical simulations. Again, for this kind of model, a single compression test is needed for the calibration, according to the process described in section 3.2 .3 The compression test $\mathrm{CH} 2$, at a confining compression of $500 \mathrm{kPa}$, has been used here for calibration (Figure 6. Clumps of type $\mathrm{C} 1$ were selected because they are the ones (among clumps $\mathrm{C} 1$ and $\mathrm{C} 2$ ) leading to a shear strength at large deformations the closest from the experimental shear strength, even if the simulated strength is still too low (i.e. more complex clumps made for instance of three particles should be considered to increase the particle interlocking).

The parameters identified for the DEM-C model are shown in Table 3 with an initial relative density of the numerical sample of $93.3 \%$ (computed with respect to the highest and smallest density numerically reachable with the REDF compaction

TABLE 2 Parameters of the model DEM-R calibrated on the Hostun sand RF from the triaxial compression CH5.

\begin{tabular}{lcccc}
\hline$\eta_{r}$ & $\begin{array}{c}\varphi_{c} \\
(\mathrm{deg})\end{array}$ & $\begin{array}{c}E_{c} \\
(\mathrm{MPa})\end{array}$ & $\alpha_{s}$ & $\alpha_{r}$ \\
\hline 0.55 & 19 & 500 & 0.3 & 5.0 \\
\hline
\end{tabular}


Model: DEM-R
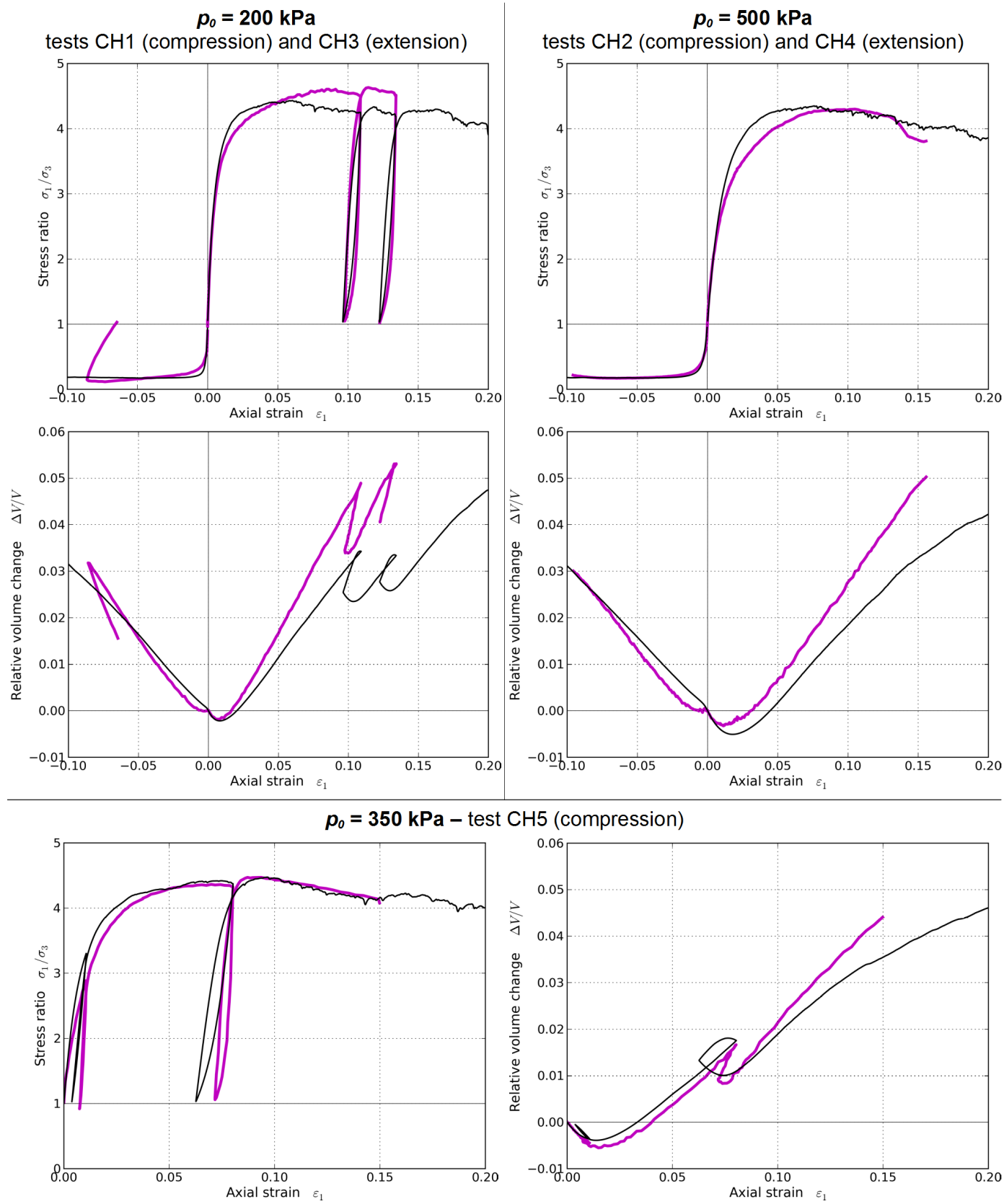

FIGURE 5 Calibration of the model DEM-R (with rolling resistance) from the drained compression CH5 (bottom) and simulated responses on other compression and extension tests.

process ${ }^{33}$ ). This high relative density identified numerically is in agreement with the initial density measured experimentally which is in the range of the highest densities for the Hostun sand.

The peak shear strength is correctly reproduced by the model however the simulated dilatancy is more important than the experimental one. The higher dilatancy of the model is consistent (as suggested by Rowe's law) with the fact that the difference between the shear strength at large deformations (i.e. at critical state) and at the peak is more important for the model than for 
the real sand. Finally, the contact stiffness has been chosen as a compromise leading to underestimating the initial macroscopic stiffness but overestimating the stiffness in the hardening regime.

Similarly to the DEM-R model, the simulations of the compression tests $\mathrm{CH} 1$ and $\mathrm{CH} 5$, with the parameters identified from test $\mathrm{CH} 2$, are also shown in Figure 6 The prediction of the soil stiffness for this two compressions is improved with respect to what was obtained for the calibration test, particularly for the test $\mathrm{CH} 5$ where both the initial macroscopic stiffness and the stiffness in the hardening regime are quite well described. Nonetheless, the peak shear strength of tests $\mathrm{CH} 1$ and $\mathrm{CH} 5$ is underestimated in particular for the test $\mathrm{CH} 1$ performed at the lowest confining pressure. As for the DEM-R model, the Coulombian friction model used at the contact scale is not suitable to describe the slight dependence of the internal (macroscopic) friction angle on the mean pressure.

\section{5 | VALIDATION ON COMPLEX LOADING PATHS}

The three models, INL2, DEM-C and DEM-R together with the two elasto-plastic models, EPIH and EPKH, will be all considered for comparison in the case of the circular stress loading path in deviatoric stress plane, presented at the end of this section. Nevertheless, monotonous and cyclic stress proportional loading paths are first considered. For the sake of concision, simulations with the INL2 and DEM-R models only are presented for the monotonous case and for the cyclic case the DEM-R model alone is considered.

\section{1 | Monotonous stress proportional loading paths}

Stress proportional paths are defined from the parameter $b$ kept constant for a given loading path:

$$
b=\frac{\sigma_{2}-\sigma_{3}}{\sigma_{1}-\sigma_{3}}=\text { cst. }
$$

with $\sigma_{1}, \sigma_{2}$ and $\sigma_{3}$ denoting the principal stresses such that $\sigma_{1}>\sigma_{2}>\sigma_{3}$. In other words, the parameter $b$ characterizes the intermediate stress with respect to the minor and major principal stresses. For instance, $b=0$ corresponds to an axisymmetric compression $\left(\sigma_{2}=\sigma_{3}\right)$; whereas $b=1$ is an axisymmetric extension $\left(\sigma_{1}=\sigma_{2}\right)$. An additional condition to $b=$ cst. should

TABLE 3 Parameters of the model DEM-C calibrated on the Hostun sand RF from the triaxial compression CH2.

\begin{tabular}{lccc}
\hline $\begin{array}{l}\varphi_{c} \\
(\mathrm{deg})\end{array}$ & $\begin{array}{c}E_{c} \\
(\mathrm{MPa})\end{array}$ & $\alpha_{s}$ & clump \\
\hline 19 & 150 & 1.0 & $\mathrm{C} 1$ \\
\hline
\end{tabular}




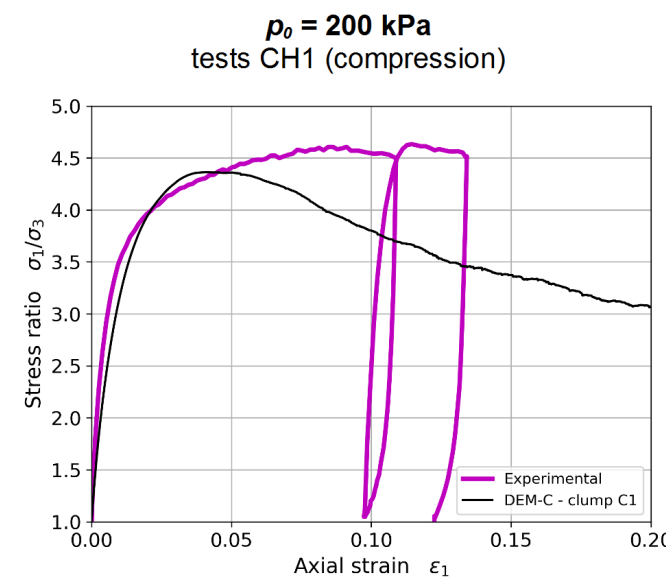

Model: DEM-C
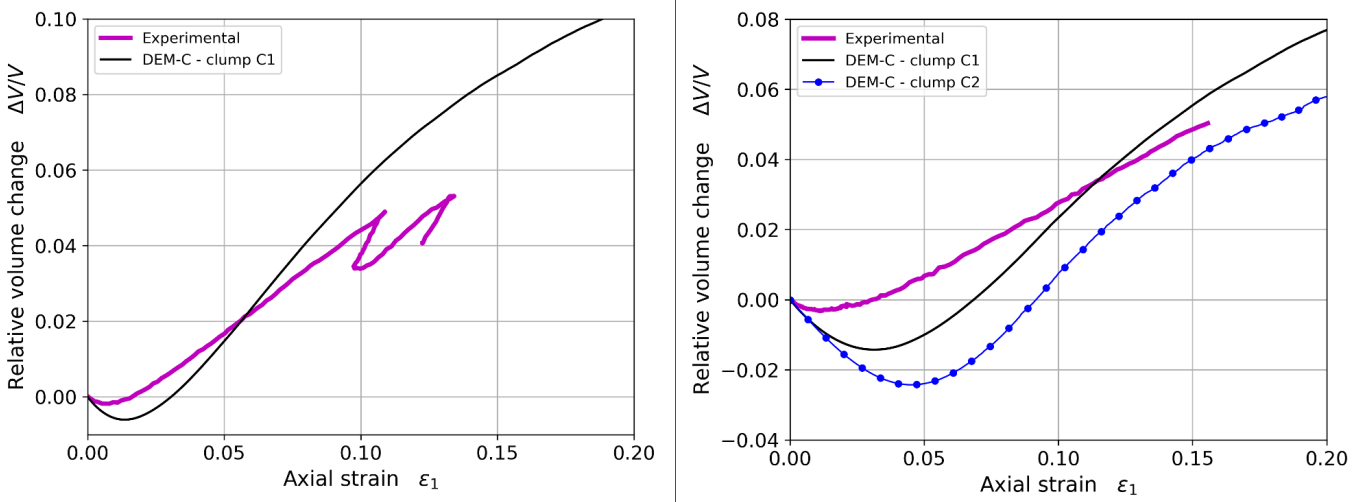

$p_{0}=350 \mathrm{kPa}-$ test $\mathrm{CH} 5$ (compression)
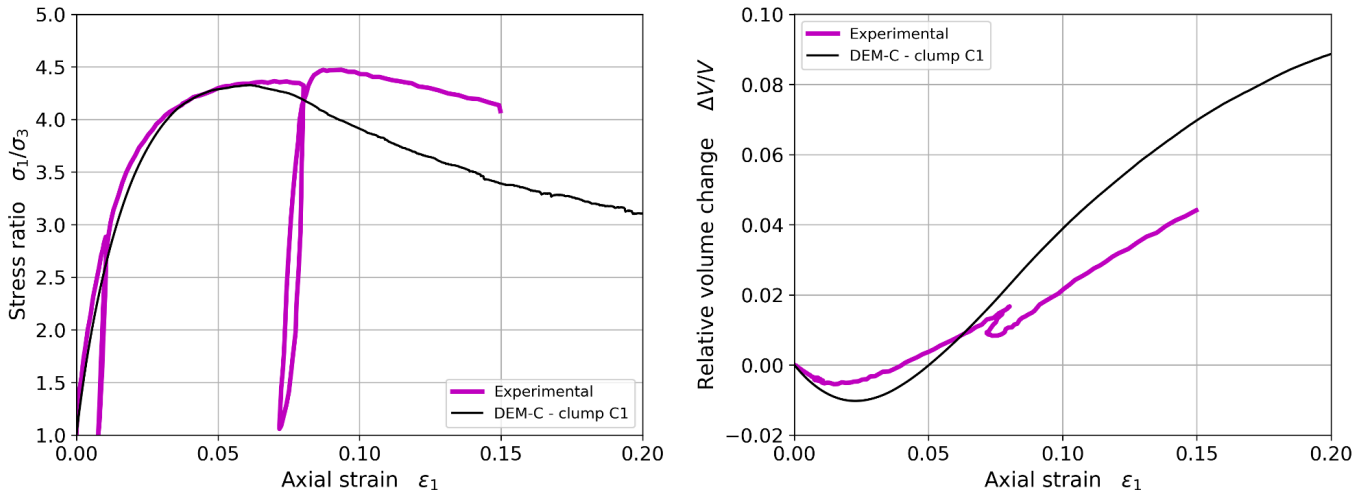

FIGURE 6 Calibration of the model DEM-C (with clumps $\mathrm{C} 1$ and $\mathrm{C} 2$ ) from the drained compression test $\mathrm{CH} 2$ (top-right) and simulated responses on other compression tests (for clump $\mathrm{C} 1$ only).

be stated to fully define the stress state. Then, either the intermediate stress $\sigma_{2}$ or the mean pressure $p$ is also fixed for a given loading path. In this section both $b$ and $\sigma_{2}$ are fixed.

The experimental and simulated tests are performed by controlling the principal strain $\varepsilon_{1}$ to impose a compression in direction ' 1 ' (i.e. $\dot{\varepsilon}_{1}>0$ ). Consequently, $\sigma_{1}$ constitutes a response parameter of the soil, $\sigma_{2}$ is kept fixed and $\sigma_{3}$ is imposed such that:

$$
\sigma_{3}=\frac{\sigma_{2}-b \sigma_{1}}{1-b}
$$


Two monotonous stress proportional loadings are considered. As resumed in Table 4 , they are both performed after an isotropic compression up to $p_{0}=500 \mathrm{kPa}$, thus $\sigma_{2}$ is fixed to $500 \mathrm{kPa}$ for the two loadings whereas $b=0.286$ for one loading and $b=0.667$ for the other.

The three principal stresses and the volumetric strains are presented in Figures 7 and 8 to compare the predictions of the INL2 and DEM-R models with the experimental data. Nevertheless, it is worth noting that $\sigma_{2}$ and $\sigma_{3}$ are imposed via the loading program and do not constitute response parameters. They are displayed in order to show the stress state is actually threedimensional (and not only axisymmetric); but experiments and models should be compared by focusing on response parameters, i.e. $\sigma_{1}$ and the volumetric strain $\varepsilon_{v}$.

The loading phase only has been simulated with the INL2 model (Fig. 7 , as the unloading was not provided for the Cleveland Workshop, whereas both loading and unloading have been described with the DEM-R model.

The stress responses $\sigma_{1}$ simulated by the two models are surprisingly very close. For $b=0.286$ (test SFHD512) $\sigma_{1}$ is overestimated by the two models INL2 and DEM-R, even if this overestimation is slightly more important for the DEM-R model. For $b=0.667$ (test SFHD514) the prediction of $\sigma_{1}$ by the two models is in good agreement with the experimental data. The simulations with the DEM-R model of the axisymmetric compression and extension at a confining pressure of $500 \mathrm{kPa}$ (test $\mathrm{CH} 2$ in Figure 5 show that the maximum shear strength is overestimated in compression whereas it is rather well predicted in extension. As the loading path for $b=0.286$ is closer to the axisymmetric compression path than the one for $b=0.667$, this could explain, at least partially, why there is an overestimation of $\sigma_{1}$ for $b=0.286$ with the model DEM-R and not for $b=0.667$.

The models INL2 and DEM-R differ mainly with respect to the volumetric deformations. The prediction given by the model INL2 is better than the one of the DEM-R model. In particular, the model DEM-R fails in describing the initial volumetric reduction for $b=0.667$. This result could have been expected as the latter path is relatively close to the axisymmetric extension path (obtained for $b=1.0$ ) for which the model misses the almost isochoric response of the sand sample at the initiation of the

TABLE 4 Characteristics of the stress proportional loading paths, the name of the loading refers to the names given to the experimental tests $\sqrt{20}$ ( ${ }^{17}$ for the names into brackets).

\begin{tabular}{lcccc}
\hline $\begin{array}{l}\text { Name } \\
\text { (Exp. test) }\end{array}$ & Type & $\begin{array}{c}\text { Initial confinement } \\
p_{0}(\mathrm{kPa})\end{array}$ & $\sigma_{2}=$ cst. or $p=$ cst. & $b$ \\
\hline SFHD512 (HH1) & monotonous & 500 & $\sigma_{2}=p_{0}=$ cst. & 0.286 \\
SFHD514 (HH2) & monotonous & 500 & $\sigma_{2}=p_{0}=$ cst. & 0.667 \\
SFHD72 & cyclic & 500 & $p=p_{0}=$ cst. & 0.667 \\
SFHD216 & cyclic & 200 & $p=p_{0}=$ cst. & 0.0 \\
\hline
\end{tabular}



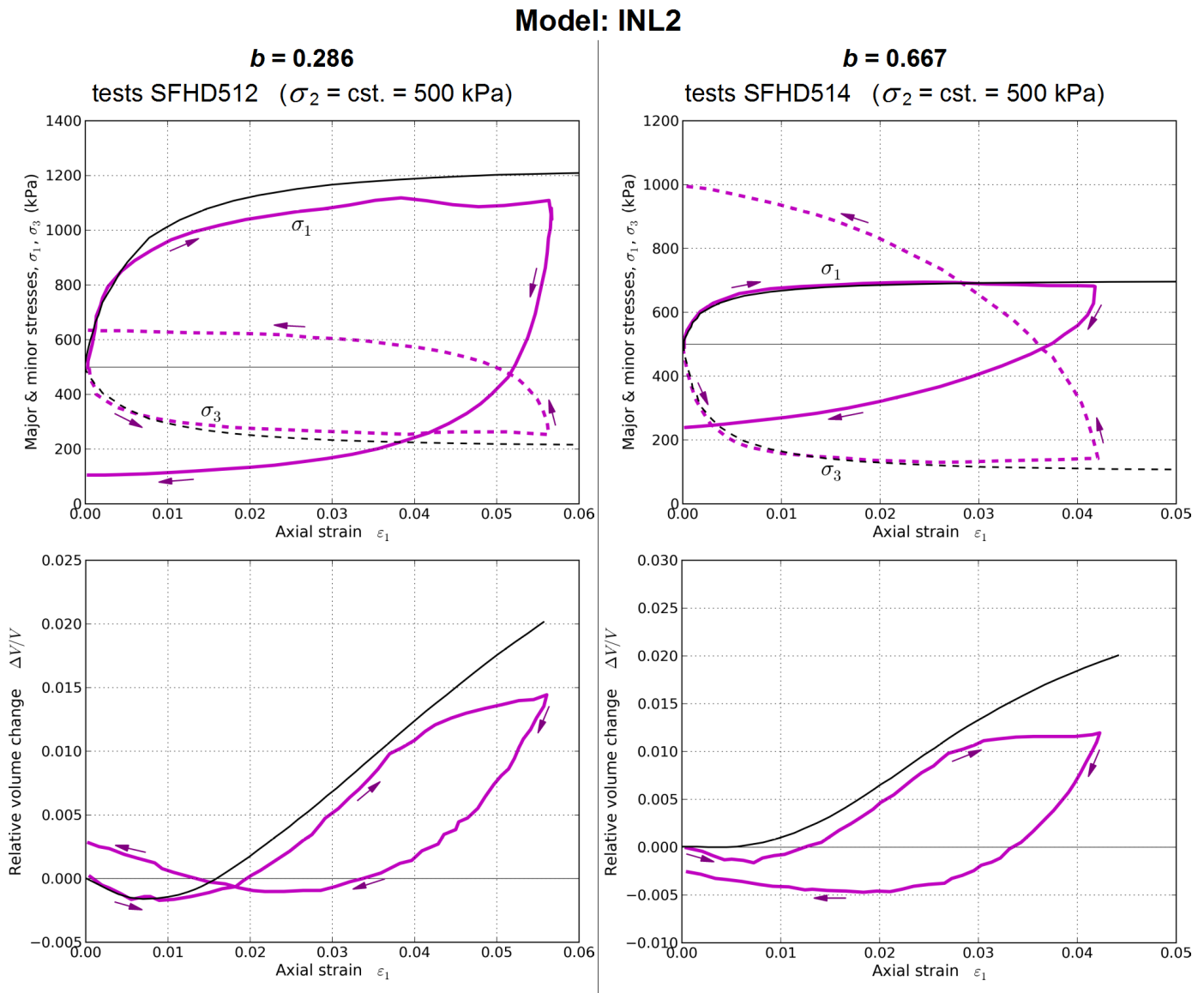

FIGURE 7 Validation of the model INL2 on stress proportional loading paths at constant intermediate stress.

extension loading (tests $\mathrm{CH} 3$ and $\mathrm{CH} 4$ in Figure 5 .

Apart from this difference, the strong reduction of the dilatancy (occurring from $\varepsilon_{1} \approx 0.042$ for $b=0.286$ and from $\varepsilon_{1} \approx 0.03$ for $b=0.667)$ is not described by the models INL2 and DEM-R. As indicated by Lanier and Zitouni $\frac{19}{19}$ this change in the volumetric strain rate is attributable to the localization of deformations occurring quite early in such tests (apparently "less stable than axisymmetric ones" ${ }^{\prime 19}$ ). By definition the model INL2 is a model of the intrinsic constitutive behavior of the soil and discards the localization of deformations. Concerning the model DEM-R, even if the localization of deformation could happen in such a numerical granular assembly, the low number of particles used here (10,000 for a 3D assembly) restrains its development. Consequently, this divergence of the models with respect to the experimental data is actually expected. Furthermore, Both model predictions are in good agreement between themselves and constitute therefore a rather trustworthy prediction of the volumetric deformation that would occur if it would be possible to delay the localization in the experimental tests. Similarly, the overestimation by the models of the axial stress initially pointed out for $b=0.286$ may not be due to a defect of models 

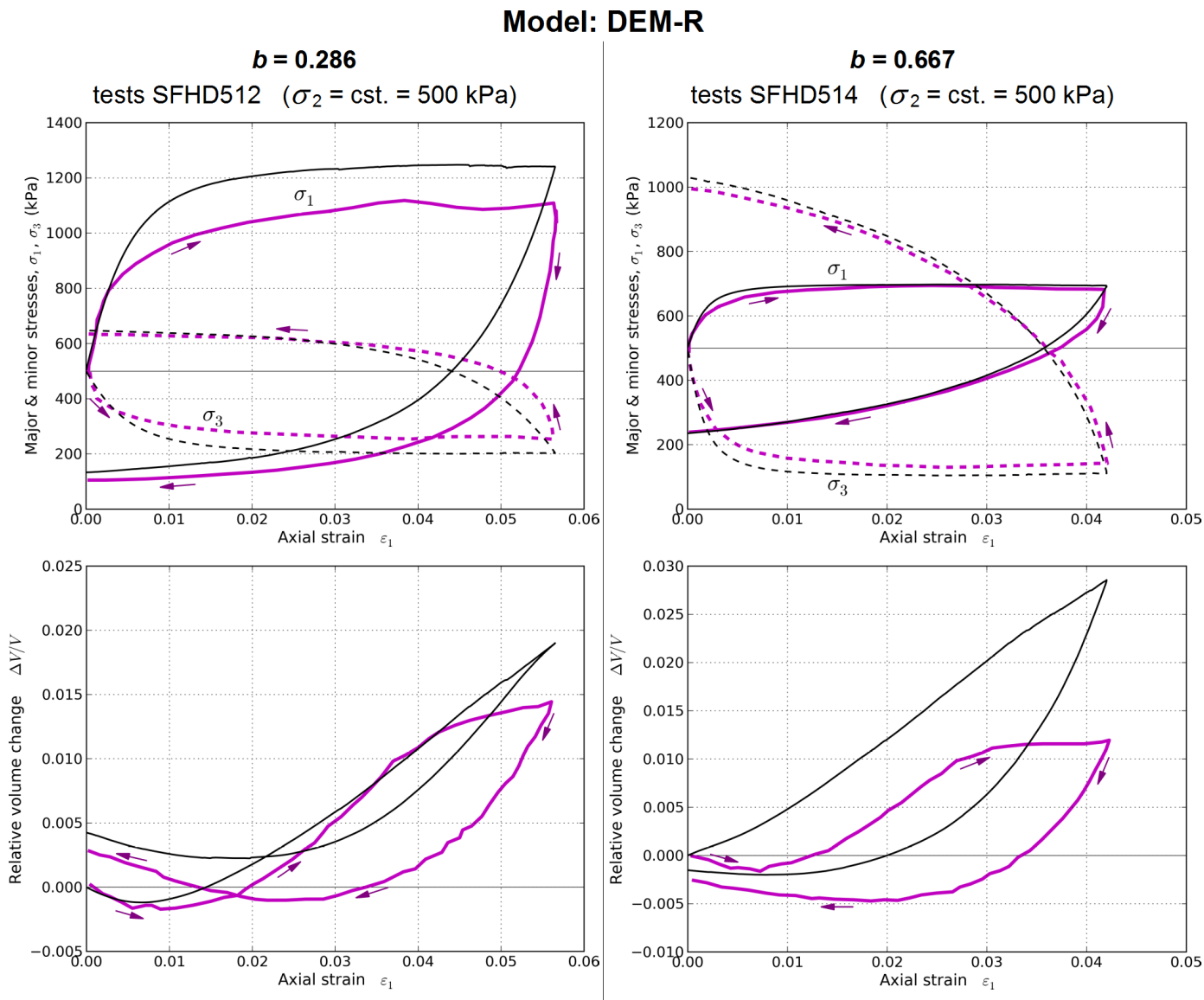

FIGURE 8 Validation of the model DEM-R (with rolling resistance) on stress proportional loading paths at constant intermediate stress.

only, but also to the localization of deformation in the experimental response taking away the measured stress states from what should be the intrinsic ones.

Finally, during the unloading phase, the major and minor principal stress directions are inverted. In these conditions, the effect on the macroscopic behaviour of the zone of localized deformations, appeared during the loading phase, could be assumed as less important during the unloading phase (unless a new localized zone develops during the unloading phase with an orientation consistent with the new principal stress directions). The shape rather regular of the experimental plots of the stresses and of the volume change for the unloading phase (contrary to the loading phase) supports this hypothesis (although it is not a proof). In the framework of this assumption, the predictions of the models can still be compared to the experimental measurements in the unloading phase; accordingly the good estimation by the model DEM-R of the residual volumetric deformation and the residual axial stress after the complete unloading is worthy of note. 


\section{2 | Cyclic stress proportional loading paths}

Cyclic loadings combined with stress proportional conditions are investigated in this section. These loading paths were not considered for the benchmark of the workshop of Cleveland. Consequently, experimental data has been extracted from the PhD thesis of Zitouni ${ }^{20}$. In the framework of phenomenological constitutive modeling, the description of the response to cyclic loadings requires constitutive relations specifically designed for that purpose and implementing particular history parameters. DEM models implicitly integrate the whole history of a loading by explicitly describing the evolution of the micro-structure induced by the loading. Therefore, it is interesting to evaluate a DEM model in such loading conditions.

As in the previous section the stress proportional condition is performed by fixing the parameter $b$ (Eq. 10). However, the additional loading condition consists here in keeping constant the mean pressure equal to the confinement reached after the preliminary isotropic compression: $p=p_{0}$. Here again, tests are performed by controlling the principal strain $\varepsilon_{1}$, consequently $\sigma_{1}$ is a response parameter and $\sigma_{2}$ and $\sigma_{3}$ are imposed such that:

$$
\begin{gathered}
\sigma_{2}=\frac{1-2 b}{b-2} \sigma_{1}+3 p_{0} \frac{1-b}{2-b} \\
\sigma_{3}=\frac{b+1}{b-2} \sigma_{1}+\frac{3 p_{0}}{2-b}
\end{gathered}
$$

Two cyclic loadings are considered, they differ from the mean pressure and the value of $b$ as detailed in Table 4 One loading with $b=0.667$ leads to a three-dimensional stress state, whereas the other one with $b=0$ is actually an axi-symmetric test with respect to direction ' 1 '. Cycle amplitude is fixed with respect to the principal strain $\varepsilon_{1}$. For both tests the amplitude of the first loading/unloading cycle is relatively important. Then the amplitude of the following cycles is reduced and kept constant such that $\Delta \varepsilon_{1}=3.8 \%$ for the test SFHD216 and $\Delta \varepsilon_{1}=4.9 \%$ for SFHD $722^{1}$

Concerning the first loading/unloading cycle, the comments previously given about the model DEM-R can be repeated here:

- underestimation of the axial stress $\sigma_{1}$ and of the dilatancy for the first loading phase of test SFHD216 $(b=0$ and $p=$ $200 \mathrm{kPa}$ ), which is actually an axisymmetric compression at a "low" confining pressure as for test CH1 (cf. Fig. 5 ;

- initial compaction of the first loading phase of test SFHD72 $(b=0.667)$ not described by the model as for test SFHD514 (Fig. 8 , which are both close to the axisymmetric extension path (see test $\mathrm{CH} 4$ in Figure 5 ;

- larger discrepancy between predicted and experimental volumetric deformation for test SFHD72 when localization of deformation occurs, as for test SFHD514 in Figure 8

\footnotetext{
${ }^{1}$ Amplitudes indicated in ${ }^{20}$ are $\Delta \varepsilon_{1}=3.75 \%$ and $\Delta \varepsilon_{1}=5.0 \%$ for SFHD216 and SFHD72 respectively, they differ slightly from the amplitudes given here and directly measured on the plots of the experimental results displayed in the same reference 20 .
} 

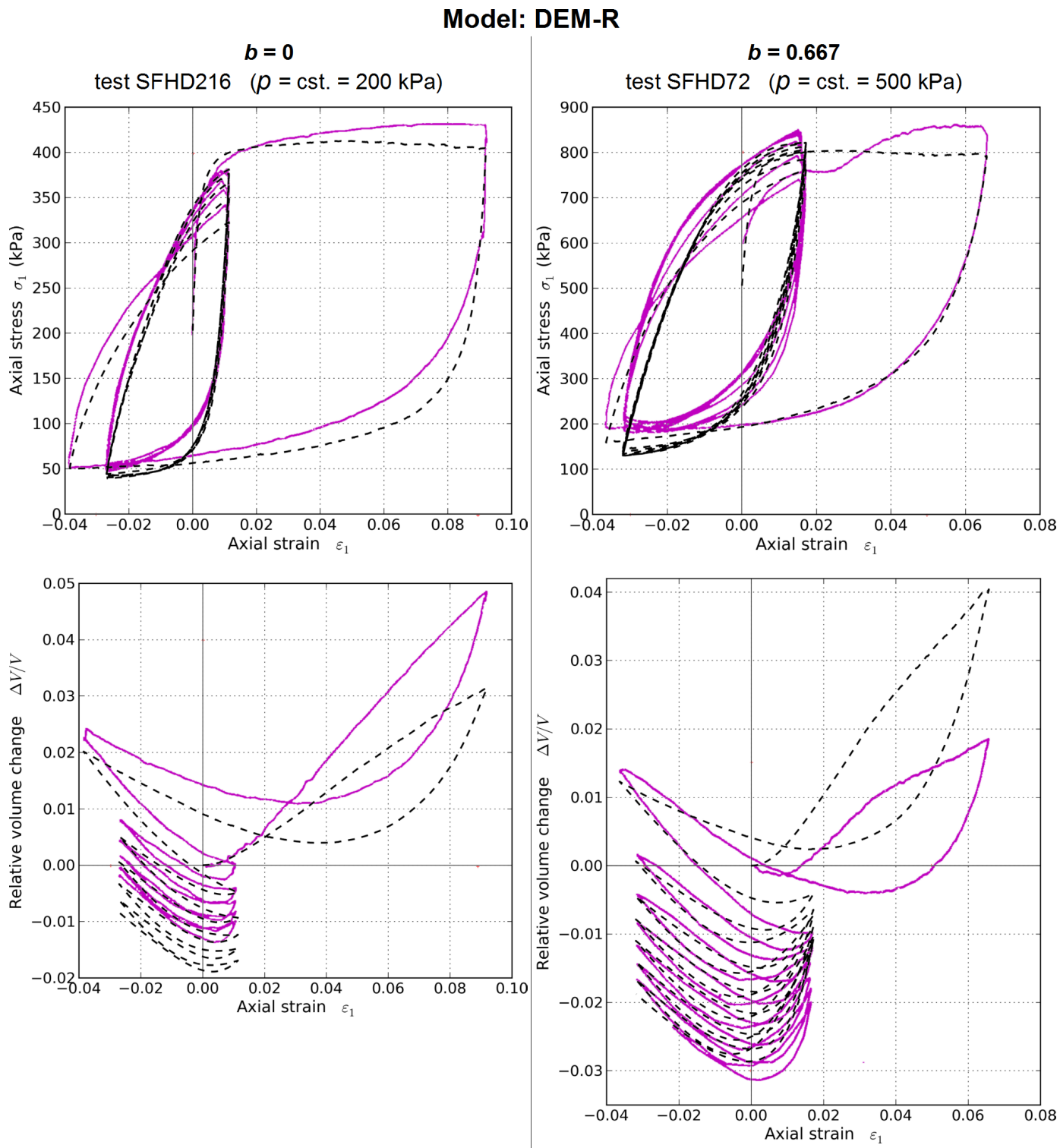

FIGURE 9 Validation of the model DEM-R on cyclic stress proportional loading paths at constant mean stress; experimental results are in continuous magenta (grey) line and simulation in dashed black line.

- good estimation of the residual stress-strain state after the first loading/unloading cycle for both $b$ values, as for tests SFHD512 and SFHD514 in Figure 8 As discussed in the previous section, this point holds for the cyclic test SFHD72 under the assumption that the localization of deformations, developed during the loading, has a negligible impact on the macroscopic response during the unloading phase (which can be still considered as essentially intrinsic). For the test SFHD216 there is no evident sign of strain localization during the first loading and this assumption could be discarded.

All these results illustrate the robustness of the discrete model with respect to the produced predictions. They are consistent from one kind of loading path to another (even if these predictions are indeed more or less good regarding the experimental results), which can be considered as a sound behaviour of the model when used in boundary value problems where loading 
paths are not known a priori at all material points.

Again, if we assume that the initial zone of localization of deformations, developed during the first loading phase for the test SFHD72, has a negligible impact on the experimental response during the next unloading phases, and also on the other reloading phases (essentially because the low strain amplitude for these other reloading phases may limit the reactivation of the initial localized zone) the model predictions can be compared with the experimental results along all the cycles for both tests, SFHD72 (8 loading/unloading cycles) and SFHD216 (6 cycles). The accordance with the experimental results is quite surprising; only the compaction during cycles for SFHD216 is overestimated, with a numerical prediction of the final volumetric strain of $-0.84 \%$ instead of about $-0.20 \%$ experimentally. Effectively, discrete models are probably today the only ones able to give such a quality of prediction for cyclic loadings (in real 3D stress conditions for test SFHD72) with only five mechanical parameters and a calibration performed from a single monotonous compression test (i.e. without any parameter calibrated on a cyclic response). Phenomenological constitutive relations need to embed ad hoc history parameters representing indirectly the evolution of the soil micro-structure. In discrete models the micro-structure is described explicitly all along the loading path (i.e. at every computational time step) which constitutes a strength, leading with few parameters to this quality of results; but also a weakness because a such explicit description of the micro-structure requires a relatively important computational cost.

Finally, the quality of the prediction may depend on the amplitude of the cycles. Here the amplitude of each cycle is quite large (up to more than 6 to $9 \%$ of deformation in the axial direction for the first cycle and then almost 4 to $5 \%$ of deformation for the following cycles); it involves at each time important changes in the micro-structure of the granular assembly and the initial state of the soil (the initial configuration of the micro-structure) is probably not the leading factor. For smaller cycles, the micro-structure may stay relatively stable and the initial state may prevail. The description of the micro-structure of a natural soil (formed under specific conditions) with a discrete model is a very difficult task (even for granular soils), whereas the change in the micro-structure induced by the mechanical loading is probably more directly taken into account by the model. Consequently, the prediction of the discrete model might have not been so good for smaller loading/unloading cycles.

\section{3 | Circular loading path in the deviatoric stress plane}

For this last type of loading path, predictions from the INL2 model and both DEM-R and DEM-C models are considered after defining the loading path in itself. These predictions will be put also in perspective with respect to the ones given by more conventional elasto-plastic constitutive relations in order to have a global view including rather fundamentally different kinds of models. 
The circular stress path in the deviatoric plane consists in continuously changing the Lode angle (which is actually the control parameter of the loading) while the mean pressure and the stress deviator are kept constant. The Lode angle $\varphi_{\sigma}$ is here defined from the axis $s_{1}$ (which is the projection of the $\sigma_{1}$ axis on the deviatoric plane).

The stress deviator intensity is characterized via the second stress invariant defined by:

$$
I_{2 \sigma}=\sqrt{\operatorname{tr}\left(\mathbf{s}^{2}\right)}
$$

where $\mathbf{s}$ is the deviatoric stress tensor. The circular stress path was carried out for $p=500 \mathrm{kPa}$ and $I_{2 \sigma}=420 \mathrm{kPa}$. Preliminary, an isotropic compression is performed up to $p_{0}=500 \mathrm{kPa}$, followed by a compression on direction '3' (i.e. $\varphi_{\sigma}=-120^{\circ}$ ) with a constant mean pressure, $p=p_{0}$, to reach the expected value of $I_{2 \sigma}$. In terms of principal stresses it means the latter is an axi-symmetric compression where $\sigma_{1}$ and $\sigma_{2}$ are imposed such that:

$$
\sigma_{1}=\sigma_{2}=\frac{3 p-\sigma_{3}}{2}
$$

while $\sigma_{3}$ is increased up to $842.93 \mathrm{kPa}$. Finally the circular stress loading is performed. It is constituted of two revolutions in the deviatoric stress plane representing an evolution of $\varphi_{\sigma}$ from $-120^{\circ}$ to $+600^{\circ}$. Then the mechanical state is fully stress controlled with principal stresses given by:

$$
\begin{aligned}
& \sigma_{1}=p+\sqrt{2 / 3} I_{2 \sigma} \cos \left(\varphi_{\sigma}\right) \\
& \sigma_{2}=p+\sqrt{2 / 3} I_{2 \sigma} \cos \left(\varphi_{\sigma}-120^{\circ}\right) \\
& \sigma_{3}=p+\sqrt{2 / 3} I_{2 \sigma} \cos \left(\varphi_{\sigma}+120^{\circ}\right)
\end{aligned}
$$

The strain response path is itself represented in the deviatoric strain plane. From a practical point of view a 2D orthonormal coordinate system was used to plot the strain response. The coordinates $(X, Y)$ representing a strain state $\left(\varepsilon_{1}, \varepsilon_{2}, \varepsilon_{3}\right)$ projected in the deviatoric plane are computed as ${ }^{35}$ :

$$
\begin{aligned}
& X=\sqrt{2 / 3} \cos 30^{\circ}\left(\varepsilon_{1}-\varepsilon_{2}\right)=\left(\varepsilon_{1}-\varepsilon_{2}\right) / \sqrt{2} \\
& Y=\sqrt{2 / 3}\left(\sin 30^{\circ}\left(\varepsilon_{1}+\varepsilon_{2}\right)-\varepsilon_{3}\right)=\sqrt{2 / 3}\left(\left(\varepsilon_{1}+\varepsilon_{2}\right) / 2-\varepsilon_{3}\right)
\end{aligned}
$$

Predictions of the three models INL2, DEM-R and DEM-C are compared with the experimental data in Figure 10 in terms of volumetric deformation and of the strain path in the deviatoric strain plane. Among the three models the best prediction is given by the model INL2 regarding both the deviatoric strain path and the volumetric deformations.

Both DEM-R and DEM-C models underestimate the deformations generated by the circular stress loading when they are projected in the deviatoric strain plane. This difference appears even as more important when the volumetric deformation is considered. The gap between simulated and experimental volumetric deformations can be split into two contributions. 


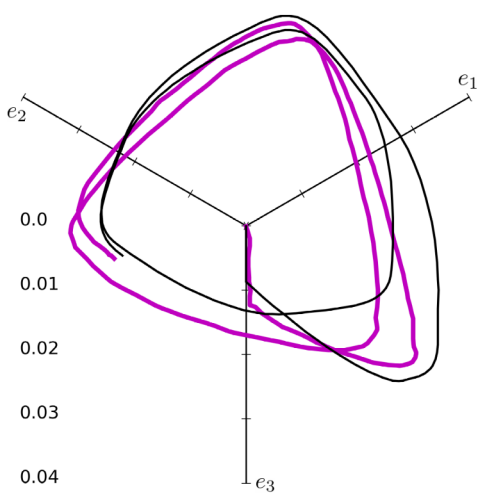

Model: INL2
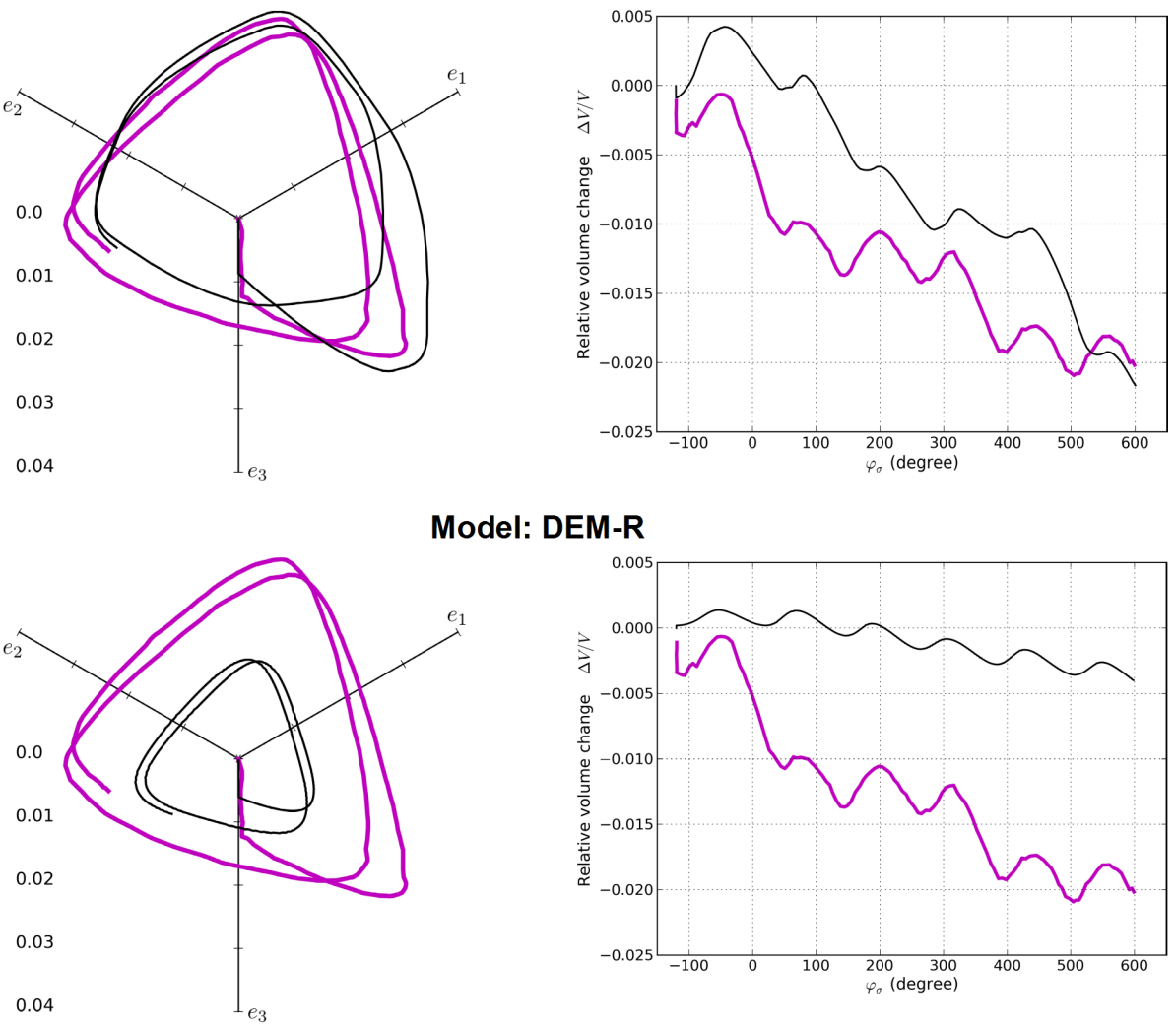

Model: DEM-R

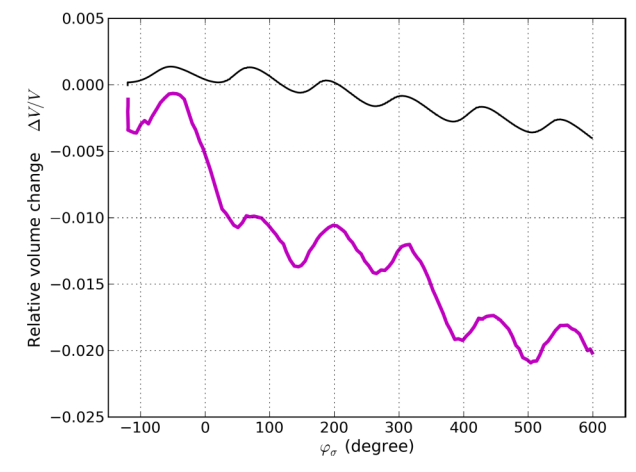

Model: DEM-C
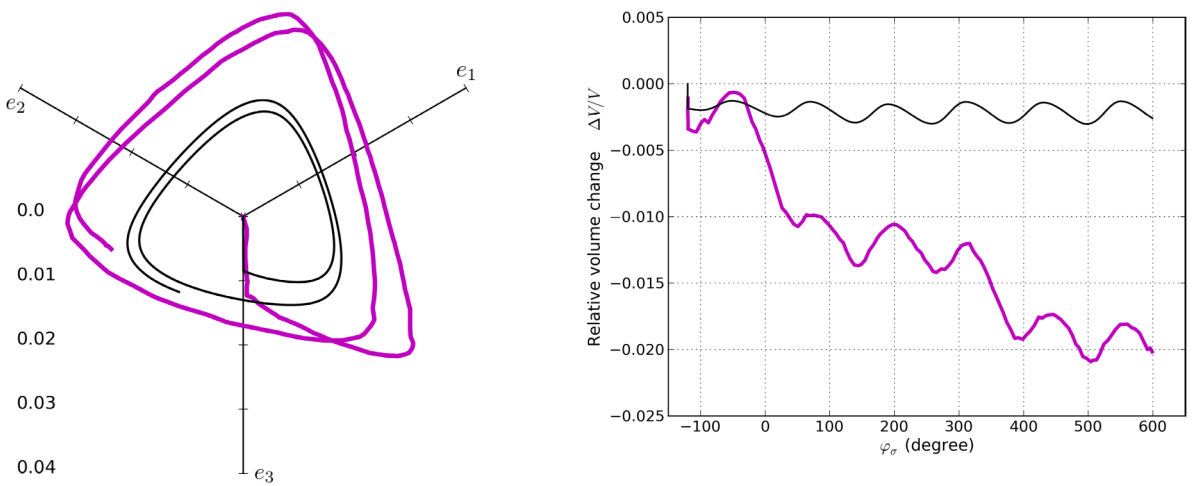

FIGURE 10 Validation of the models INL2, DEM-R and DEM-C on the circular stress loading path, the strain response in the deviatoric strain plane is displayed on the left and the volumetric response in terms of the Lode angle $\varphi_{\sigma}$ on the right.

First, experimentally the sand sample compacts, during the initial compression on direction ' 3 ' (i.e. at constant Lode angle $\varphi_{\sigma}=-120 \mathrm{deg}$ ) and at constant mean pressure, down to a relative volume change of $-0.34 \%$. Second, irreversible volumetric deformations progressively accumulate during the circular stress loading to reach at the end of the test a total volume change of $-2.0 \%$. The first compaction during the initial compression is predicted, but underestimated, by the model DEM-C, whereas the model DEM-R fails to describe it, showing a compression occurring almost at constant volume (although the discrete model actually deforms during this phase, as it is visible in the deviatoric strain plane, but in an almost purely deviatoric way). Inversely, the DEM-R model presents irreversible volumetric deformation during the circular loading, but not as large as the 


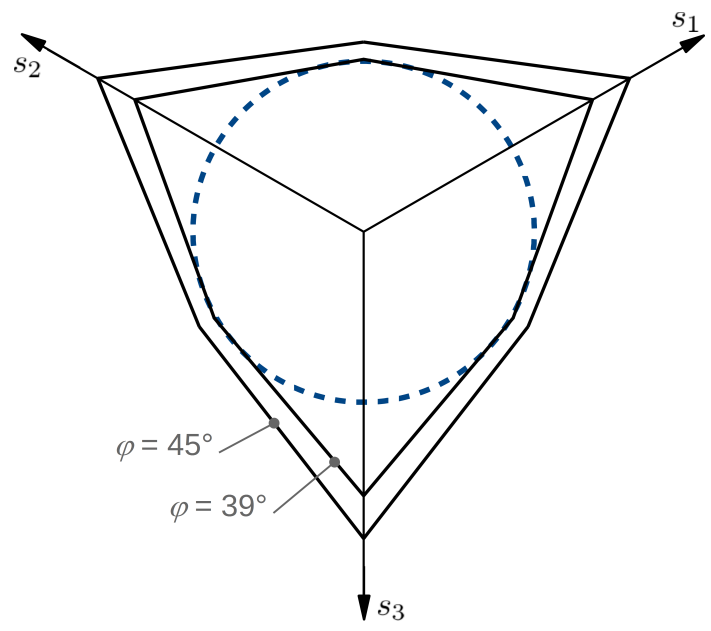

FIGURE 11 Mohr-Coulomb criteria (for internal friction angle of $39^{\circ}$ and $45^{\circ}$ ) and the circular stress loading path in the deviatoric stress plane.

experimental one, while rather reversible volumetric deformation of low amplitude are described with the model DEM-C.

The Mohr-Coulomb criteria for internal friction angles of $39^{\circ}$ and $45^{\circ}$ (representing the failure envelopes of the dense Hostun sand in compression and extension respectively) are compared in Figure 11 with the imposed circular stress path in the deviatoric stress plane. The circular stress path stays included within the Mohr-Coulomb limit condition, quite far from the latter in the compression directions $\left(\varphi_{\sigma}=-120 ; 0\right.$ and $\left.120^{\circ}\right)$ and very close to the criterion in the directions of extension $\left(\varphi_{\sigma}=-60 ; 60\right.$ and $180^{\circ}$ ). Therefore, the soil sample is somehow subjected to loading and unloading cycles in the hardening zone and particularly quite far from the maximum shear strength in the compression directions. The main weakness of the DEM-R and DEM-C models highlighted in axi-symmetric conditions (see Figures 5 and 6 is the overestimation of the stiffness in the hardening regime, resulting in underestimating the deformations induced by the circular stress loading (which is stress controlled by definition).

To resume the models DEM-R an DEM-C involve few mechanical parameters but in return there is no parameter to fit directly the stiffness of the simulated response in the hardening regime. From another side, the stiffness in the hardening regime for the INL2 model depends directly on one parameter (among the 16 constitutive parameters of the model) and the simulated responses fit closely the experimental results for the axi-symmetric compressions and extensions paths (Figure 4 . In the same way, the principal strains resulting from the circular stress loading are closely predicted (Figure 12 .

To assess the relevance of the discrete numerical models with respect to constitutive relations more classically used in an engineering context, the predictions to the circular stress loading with two elasto-plastic models are considered below. These predictions were also extracted from the Cleveland's Benchmark. The first elastoplastic model ${ }^{36}$ includes a single yield (or 

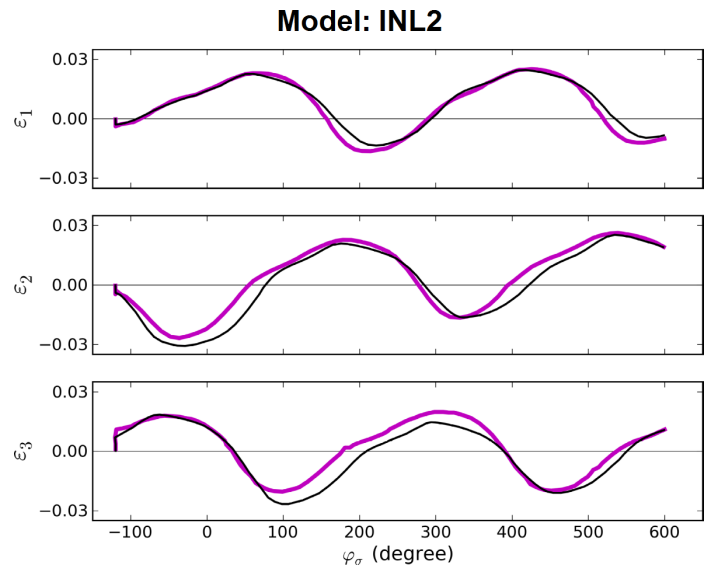

Model: DEM-R
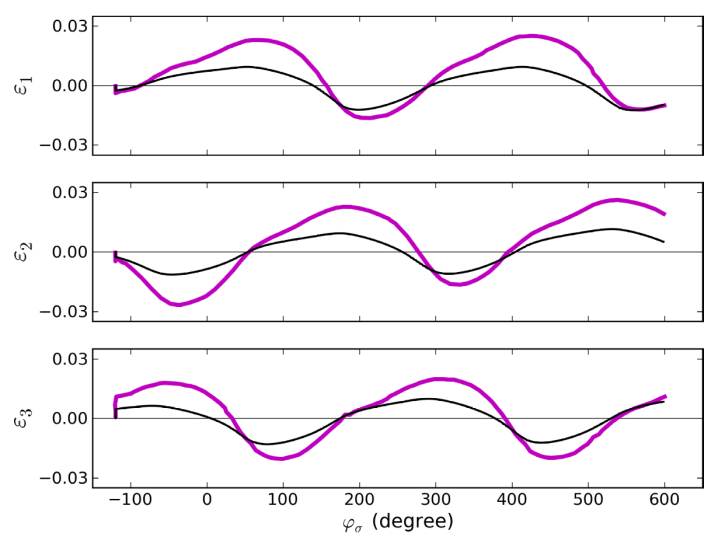

Model: DEM-C
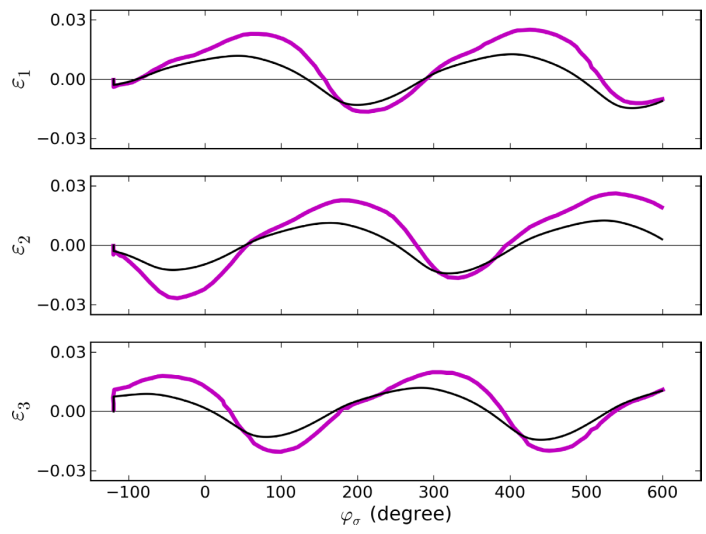

FIGURE 12 Response of the models in terms of principal strains to the circular stress loading path.

bounding) surface associated with an isotropic hardening mechanism (EPIH model); whereas the second model ${ }^{[37}$ implements two yield surfaces, one related to an isotropic hardening mechanism and the second to a kinematic hardening mechanism (EPKH model). Despite this difference, a non-associated flow rule is considered for both models and they require almost the same number of constitutive parameters (11 and 12 parameters for the EPIH and EPKH models respectively). The simulated responses are shown in Figure 13 


\section{Elastoplastic models}
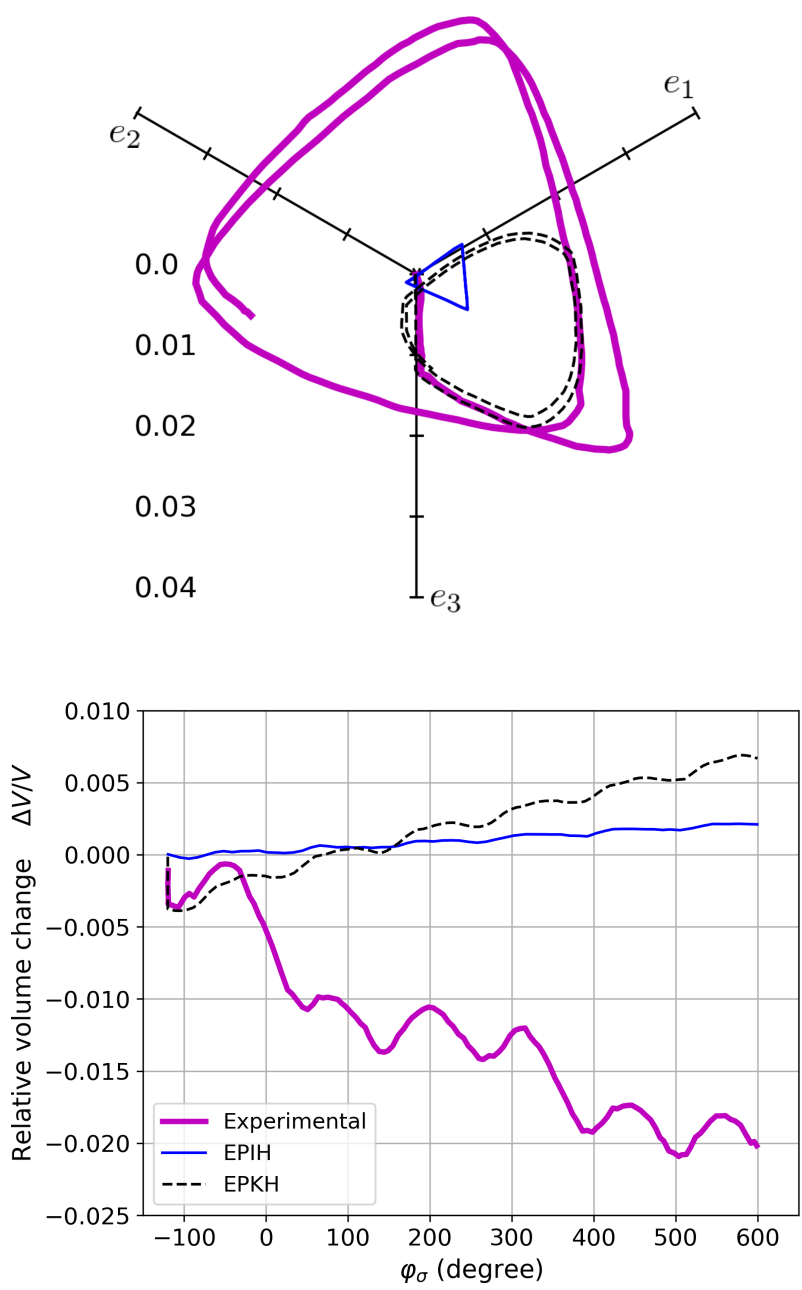

FIGURE 13 Elastoplastic constitutive relations: simulated response to the circular stress loading path for an isotropic hardening mechanism (EPIH model, after Saleeb and Lou ${ }^{36}$ ) and a kinematic hardening (EPKH model, after Cambou and Jafari ${ }^{37}$ ) in addition to the isotropic one.

The EPIH model does not lead to any noticeable deformation during the initial compression at constant mean pressure contrary to what was observed experimentally. According to the authors ${ }^{36}$ it is the consequence of a too high shear elastic modulus (which would implicitly means the deformation during this first phase is essentially elastic). Then deformations induced by the circular stress loading are largely underestimated, no hysteresis is described concerning the deviatoric strain and a very slight volume increase is predicted instead of a global compaction. The analysis of the experimental data ${ }^{19}$ shows the irreversible compaction of the sand sample occurs around the directions of compression $\left(\varphi_{\sigma}=-120,0,120, \ldots\right.$, deg $)$ whereas the dilatancy is observed in the vicinity of the directions of extension $\left(\varphi_{\sigma}=-60,60,180, \ldots\right.$, deg $)$. Consequently, the EPIH model fails to describe the global compaction probably because the stress path stays within the yield surface, avoiding the development of plastic deformations, particularly for the directions of compression where a plastic compaction would be expected. 
Concerning the EPKH model, the prediction of the deviatoric and volumetric strains during the initial compression phase at constant mean pressure is excellent (even better than with the INL2 relation). Similarly, predictions are also very good at the very beginning of the circular loading up to a Lode angle of about $-90^{\circ}$. In other words, as long as the Lode angle is nearly fixed the model give a very good prediction but then deformations are underestimated and a dilatant volumetric behaviour is predicted instead of a contractive one. Cambou and Jafari ${ }^{37}$ explained that the yield surface was pushed in the direction of $s_{3}$ (i.e. the direction of compression) during the initial compression. Then plastic strain develops at the beginning of the circular loading; but, at a given point, the stress path points inward the yield surface and the simulated response turns into an elastic one limiting the plastic compaction of the medium.

Putting the simulations with the discrete element models in perspective with respect to the two last ones based on elastoplastic models shows that, although the predictions with the DEM on the circular path is not quantitatively totally right, they are generally safer than those from the elastoplastic models (which are actually advanced elastoplastic constitutive relations). Probably because all the possible irreversible mechanisms at the grain scale implicitly taken into account with the DEM cannot be described via some few yielding surfaces. Increasing the number of yielding surfaces and the related hardening mechanisms (requiring each time additional parameters to be defined) would improve the prediction, but for very high number of yielding surfaces it will actually tend towards a fully incrementally non-linear relation as the INL2 model.

The quality of the prediction of the discrete element models in the hardening regime could eventually be improved thanks to a more thorough description of the soil micro-structure (grain shape) and its initial state (fabric and connectivity). However, this would imply more sophisticated models and sample generation processes ${ }^{30138}$ more heavy to implement for a routine use. An example is given here with the DEM-R model for which the connectivity of the initial granular assembly has been chosen such that the model presents a stiffness in the hardening regime closer to the experimental one. All the mechanical parameters of the model identified in the initial calibration were kept the same (Table 2 and the initial sample density is also unchanged. Initial states of the granular assembly at a fixed density and different coordination numbers (i.e. different average numbers of interactions per particle) were achieved by adding an inter-particle adhesion during the isotropic compaction of the numerical sample. This inter-particle adhesion is then removed at the end of the isotropic compaction.

The "new" calibration of the model with an initial coordination number of 2.23 (instead of 3.35 for the initial calibration) is presented in Figure 14 Thanks to this improvement the experimental stress-strain response in the hardening regime is now well described. The simulated response to the circular stress loading path with this new calibration is compared with the initial "blind prediction" in Figure 15 As the stiffness in the hardening regime has been reduced with the new calibration, thanks to a lower initial connectivity, the deformations generated by the circular stress path are slightly more important. Hence the change brought by the new calibration is in the right direction, but this improvement is not enough to reproduce closely the strain path 
Model: DEM-R
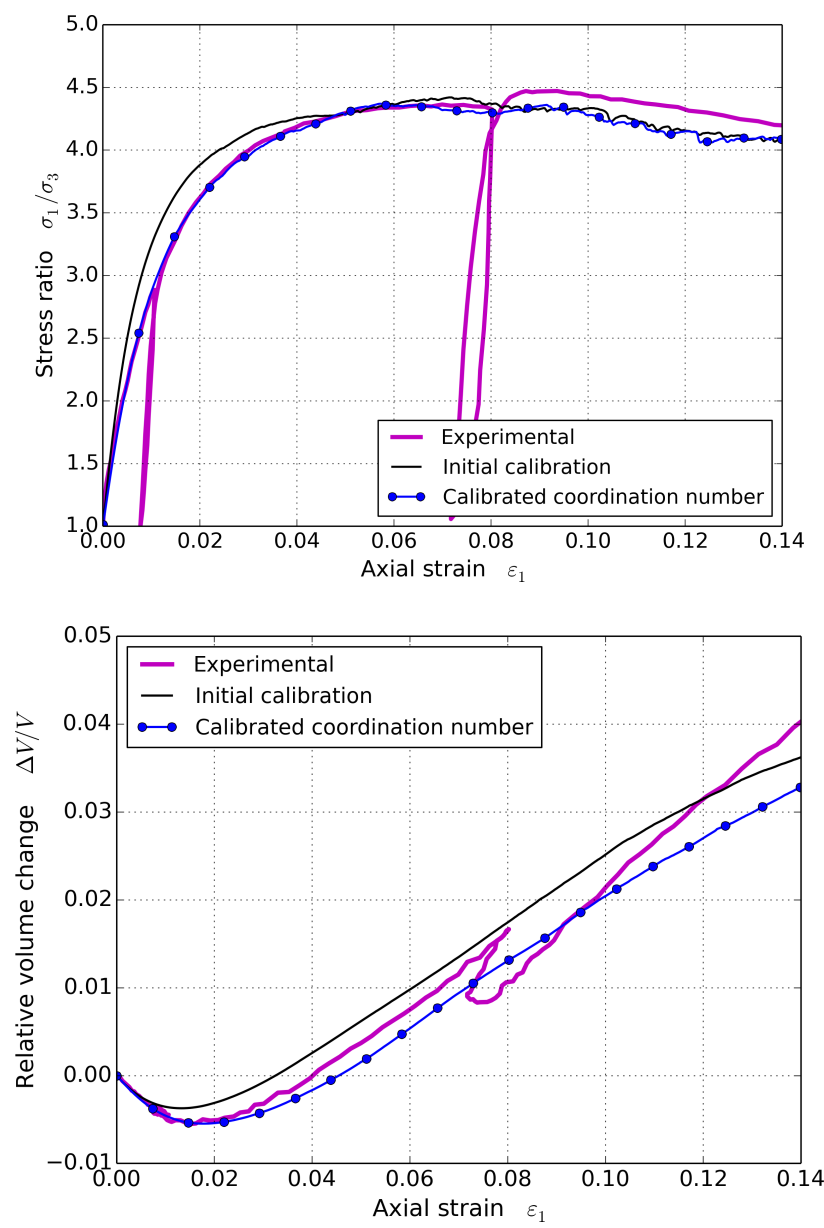

FIGURE 14 Calibration of the initial coordination number for the DEM-R model with respect to the axisymmetric compression test $\mathrm{CH} 5$, the initial calibration of the model is also shown for comparison.

in the deviatoric strain plane, as well as the volume change.

To investigate other possibilities for improving the prediction on the circular stress loading path with the discrete models a short parametric study is presented in the following. Moreover, in order to stay within the scope of this paper, considering the rather simple numerical models DEM-R and DEM-C as defined so far, the calibration of the coordination number as discussed previously is discarded. Only some contact parameters, and the initial sample density are concerned by this parametric study.

There is no constitutive parameter for the DEM-R and DEM-C models independently related to the stiffness of the stress-strain response in the hardening regime. Nevertheless, some mechanical parameters or the initial density can impact this behaviour but they will also affect other mechanical properties. For instance the contact friction angle will impact the hardening behaviour but also the maximum shear strength and the dilatancy, as the initial density; or the contact stiffness will also affect the initial 
Model: DEM-R
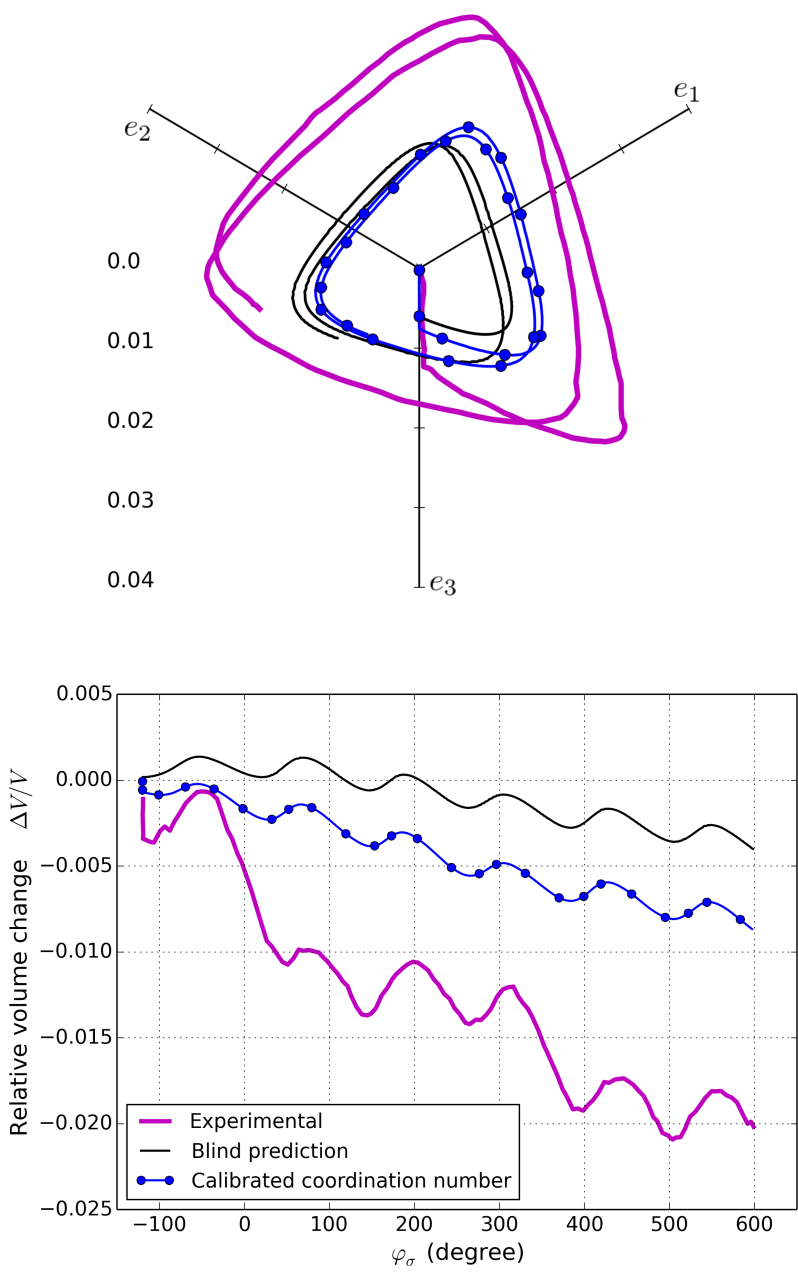

FIGURE 15 Simulation of the circular stress path with the DEM-R model for which the coordination number at the initial state has been calibrated; comparison with the previous "blind prediction" (performed with the initial calibration) and the experimental result.

macroscopic stiffness. Consequently, the improvement of the simulated response in the hardening zone through such a parametric study will necessarily result in a degradation of the prediction of other macroscopic mechanical properties.

For the DEM-R model a slight increase of the initial porosity ( $n=0.369$ instead of $n=0.359$, line "looser state" in Fig. 16 or a slight reduction of the contact friction angle $\left(\varphi_{c}=17^{\circ}\right.$ instead of $\varphi_{c}=19^{\circ}$, line "smoother contacts" in Fig. 16 are sufficient to almost correct the too low deformations predicted by the "blind prediction" as the model is globally less stiff. However, as expected the initial calibration with respect to the axisymetric compression test $\mathrm{CH} 6$ has been degraded: the maximum shear strength and dilatancy are now underestimated as shown in Figure 17.

A similar trend is observed with the model DEM-C as shown in Figures 18 and 19 A looser initial state (relative density of $73.3 \%$ instead of $93.3 \%)$, or smoother contacts $\left(\varphi_{c}=16^{\circ}\right.$ instead of $\left.\varphi_{c}=19^{\circ}\right)$, or even softer contacts $\left(E_{c}=100 \mathrm{MPa}\right.$ instead of $E_{c}=150 \mathrm{MPa}$ ) result in larger deviatoric deformation. However, in the case of this model a contractive behaviour 

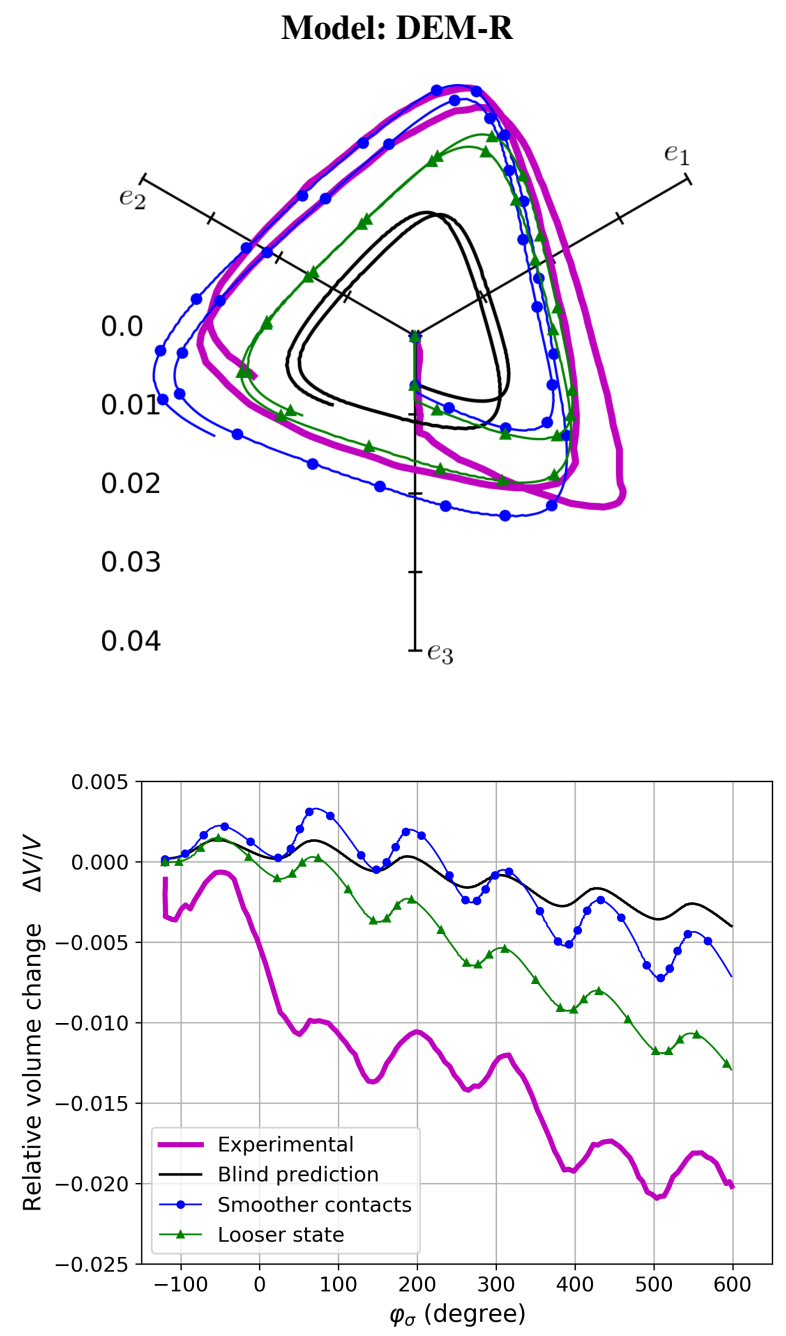

FIGURE 16 Comparison of the simulated responses to the circular stress loading with the DEM-R model for the parameters calibrated from test $\mathrm{CH} 6$ (blind prediction) and for a looser initial state or smoother contacts.

(as observed experimentally) was never reached, and even a globally dilatant behaviour is found in the "smoother contacts" case. The latter case is the one which has led to the more important shear strength reduction (Figure 19 and consequently the circular stress loading is more prone to activate an irreversible dilatant response occurring beyond the characteristic state.

A final attempt is carried out with the DEM-C model by using the clump C2 instead of C1 (Figures 3 \& \&), corresponding to a reduction of the clump elongation by a factor 2. As the elongation affects the overall macroscopic mechanical properties, the calibration of the other parameters of the model from test $\mathrm{CH} 2$ have been repeated by taking into account this new clump shape. The calibration result is presented in Figure 6 and the new values of the model parameters are: $\varphi_{c}=38^{\circ}, E_{c}=100 \mathrm{MPa}$ and $\alpha_{s}=1$ for an initial relative density of $34.5 \%$. With this set of parameters the response to a drained compression is very similar to the one given by the parameters used for the "blind prediction" excepted the shear strength at large deformation which 
Model: DEM-R
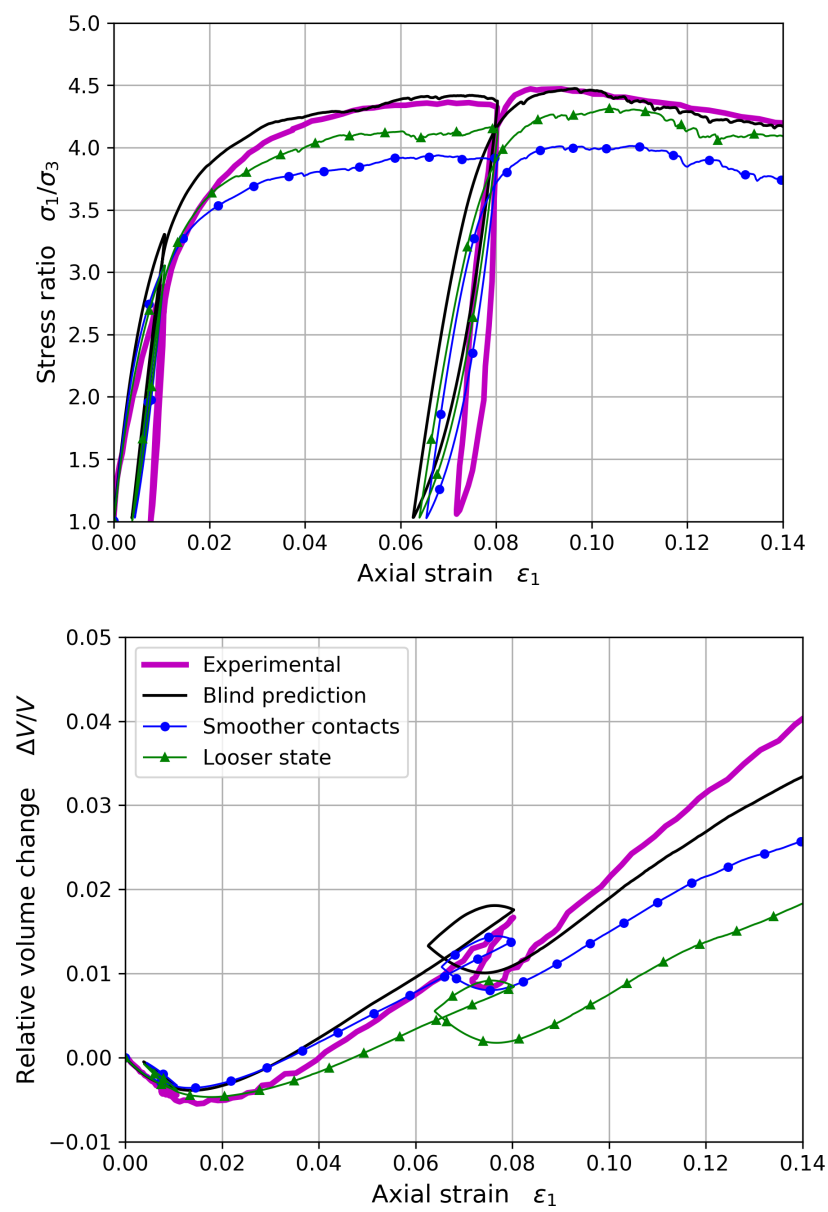

FIGURE 17 Simulations of the calibration test CH6 with the DEM-R model for a looser initial state and smoother contacts than the ones used for the blind prediction.

depends on the clump elongation only and cannot be corrected from the other model parameters. As the pre-peak behaviour is rather the same, the simulated response to the circular stress loading path with clump C1 ("blind prediction") and C2 are very close (Figure 20 . In other words, as the clump elongation is directly related to the critical state it cannot really affect the simulated response to the circular stress loading which is mainly fixed by the mechanical behaviour in the hardening regime and close to the maximum shear strength.

This parametric study shows that a change in the parameters of the models (DEM-R or DEM-C) leading to a reduction of the stiffness in the hardening regime, and accompanied by a reduction of the maximum shear strength, make possible a better prediction of the strain response to the circular stress loading. This is at least true in the deviatoric strain plane where some of the numerical predictions are even very good. On the contrary, a reduction of the stiffness in the hardening regime only (i.e. without a reduction of the maximum strength), as obtained by tuning the sample connectivity at the initial state, improves slightly the 
Model: DEM-C
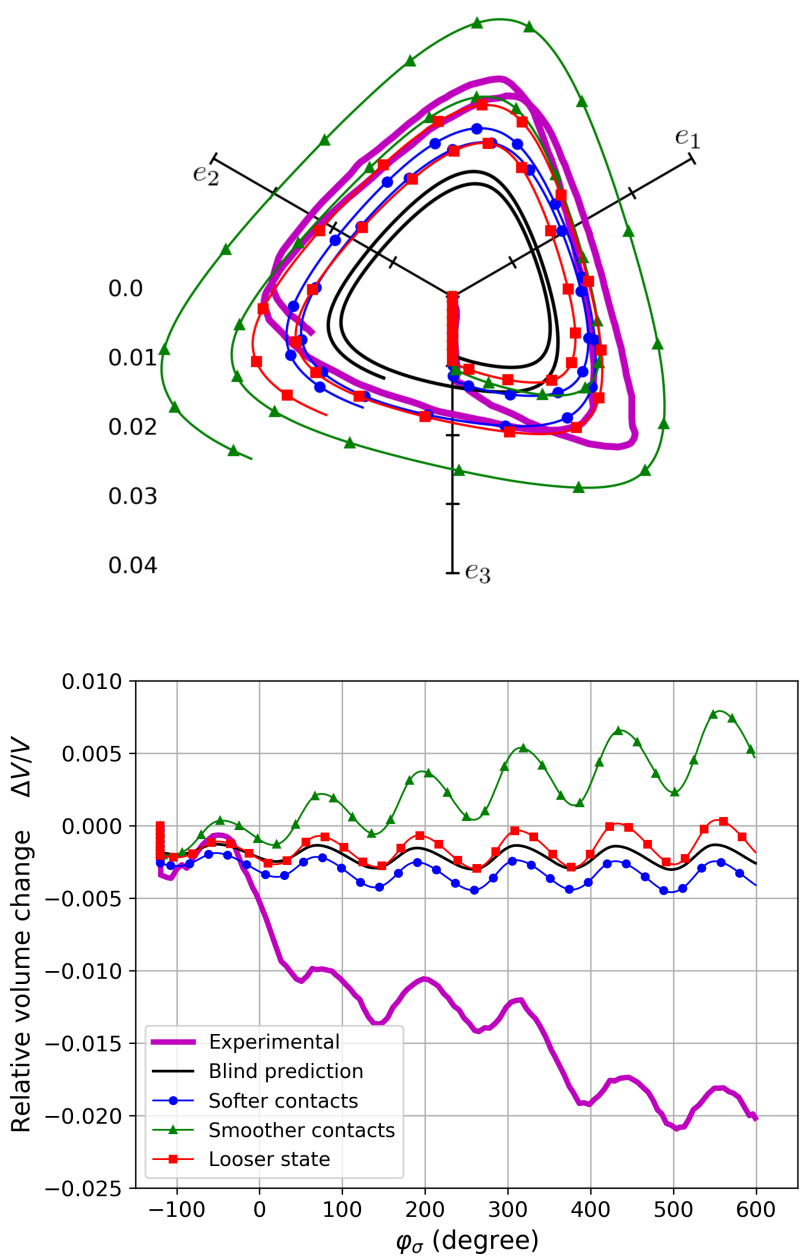

FIGURE 18 Comparison of the simulated responses to the circular stress loading with the DEM-C model for the parameters calibrated from test $\mathrm{CH} 2$ (blind prediction) and for a looser initial state, smoother contacts, and softer contacts respectively.

prediction of the strain response but was not enough to reach a really satisfying result. A conclusion from these results could be that there is experimentally a progressive degradation of the contact properties between sand grains along the circular stress loading performed at a mean pressure of $500 \mathrm{kPa}$. Such a degradation would result in a slight reduction of the maximum shear strength at the macroscopic scale. It could also participate to the important irreversible reduction of volume occurring when the stress path is around the directions of compression (i.e. for $\varphi_{\sigma}$ close to $-120 ; 0$ and $120^{\circ}$ ) that the models (DEM-R, DEM-C, but also the elasto-plastic relations) fail to describe correctly. Hence a better description of the initial state of the sand sample would not be the only improvement to bring to the DEM-R and DEM-C models. Nevertheless, without available experimental data concerning the sand after the circular stress loading, the question of the degradation of the sand grains stays open. 
Model: DEM-C
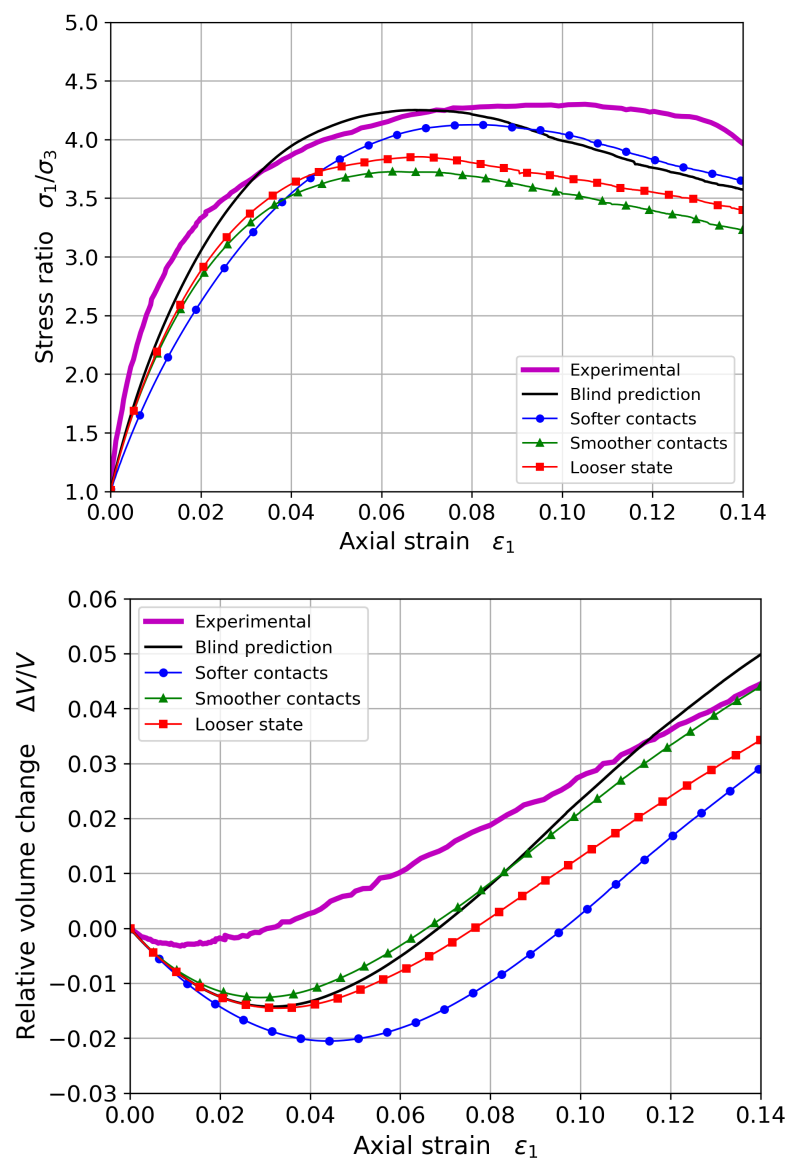

FIGURE 19 Simulations of the calibration test $\mathrm{CH} 2$ with the DEM-C model for a looser initial state, smoother contacts, or softer contacts than the ones used for the blind prediction.

\section{6 | CONCLUDING DISCUSSION}

Two discrete element models were considered in this paper, one implementing clumps made of two spherical particles (DEMC), the other considering a resistance to the rolling at the contact point between spherical particles (DEM-R). As illustrated in the section 5.3 there is no strong differences in the quality of the prediction (in both qualitative and quantitative points of view) between these two models. Nevertheless, the prediction ability of the DEM-R model may be considered as slightly more refined, probably because of the too simple clump shape considered for the DEM-C model resulting in a too high rotation of clumps around their axis of symmetry (see Figure $3 \&$ d). The three-dimensional angular shape of the Hostun sand, used here as reference, may be better taken into account with a contact law including a resistance to rolling, even if it can be viewed as a less direct (or more phenomenological) approach than the use of clumps. Despite this difference, the comments given here after holds for both types of models. 

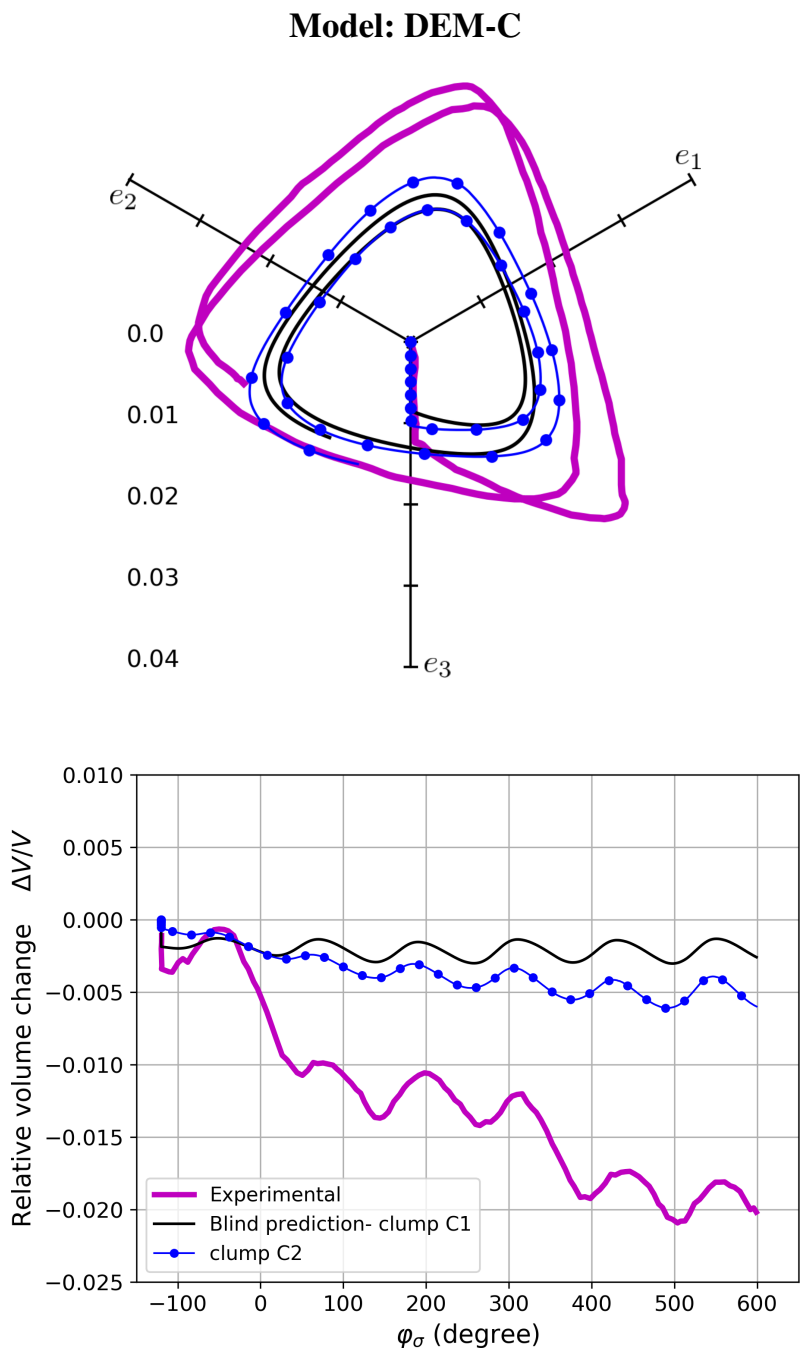

FIGURE 20 Comparison of the simulated response with the model DEM-C to the circular stress loading with two different clump elongations.

A strong advantage of the discrete numerical models is their good prediction ability in the range of large deformations for monotonous or cyclic loadings (at least for a low number of cycles since the case of numerous cycles -hundreds or more- has not been checked in this study), together with a calibration process requiring a single compression test. In such cases the loading history play an important role and it is implicitly embedded in the model. The explicit description at any loading step of the micro-structure and of the inter-particle interactions constitutes a memory of the previous loading (as it does in real granular material), and ad hoc history parameters as defined in phenomenological relations are useless.

In return, the simulated response for low deformations and monotonous loadings may depend on the initial micro-structure. It can constitute a drawback in the sense it may not be straightforward to simulate the process (followed in laboratory to create the soil sample, or resulting of the natural soil sedimentation) leading to the initial state of the soil. Besides, the possible degradation of the particles of the soil under a relative high stresses, is completely discarded by the considered models. In 
this paper the simulations of the deformations for the circular loading stress path constitute an illustration of the limitations of discrete models in these conditions. Another illustration of the drawback related to the initial state can be found in Aboul Hosn et $a l .{ }^{[29}$ where constitutive simulations with the DEM-R model have been compared with mechanical tests performed on very loose sand samples (created by moist tamping and with an initial relative density of $20 \%$ ). The validation of the model on loading paths, different from the calibration paths, were far to be conclusive. This was assumed to result from the very particular sand micro-structures (made of macropores ${ }^{40 \mid 41}$ ) obtained with the moist tamping technique and not suitably represented through the generation of the numerically sample.

Consequently, it is not because the discrete numerical model appears in this paper as rather efficient to describe the mechanical constitutive behaviour of an initially dense Hostun sand, that the model can be expected efficient as well for other granular soils, or even the same Hostun sand but with a different initial micro-structure. Of course, numerical preparation methodologies simulating a micro-structure closer to the real one could be implemented, however this departs from the objective in this paper to keep the models and their implementations rather simple for a possible use in an engineering context.

To resume, for initially dense granular soils, discrete numerical models should present a good quantitative prediction of the mechanical response in particular at large deformations (for instance to describe the failure and some complex mechanisms related to the ultimate state behaviour of a structure) or even in cyclic conditions. If in addition only few information is available to calibrate the model (for instance a single drained compression or even a single direct shear test) discrete models could even be the only model reasonable to consider for prediction on complex loading paths. For low deformations (as occurring typically when structures are designed with respect to serviceability limit state criteria) and monotonous loadings, classical non-associated phenomenological constitutive relations where the hardening regime can be adjusted with ad hoc parameters (as elasto-plastic constitutive relations) could be preferable to a discrete numerical method. The predictions of such constitutive relations in these conditions can be reasonable, with probably a more straightforward use and representing for sure a much lower computational cost. Nevertheless, as soon as a significant part of the soil body is subjected to non-proportional loading paths (i.e. not rectilinear, as the circular stress loading path considered in this paper), a conventional elasto-plastic relation may produce wrong material responses. On the other hand the discrete element method will very probably always give reasonable responses and more physically acceptable results, since the most significant physics is taken into account in this method.

\section{References}

1. Dell'Isola F., Sciarra G., Vidoli S.. Generalized Hooke's law for isotropic second gradient materials. In: The Royal Society; 2009. 
2. H.B. Mühlhaus. Application of Cosserat theory in numerical solutions of limit load problems. Ingenieur-Archiv. 1989;59(2):124-137.

3. Pijaudier-Cabot G., Bazant Z.. Non-local damage theory. Journal of Engineering Mechanics. 1987;113(10):1512-1533.

4. Darve F., Nicot F.. On incremental nonlinearity in granular media. Phenomenological and multi-scale views (Part I). International Journal For Numerical and Analytical Methods in Geomechanics. 2005;29(14):1387-1410.

5. Darve F.. Geomaterials, Constitutive Equations and Modellingch. The expression of rheological laws in incremental form and the main classes of constitutive equations, :123-148. Elsevier Applied Sciences 1990.

6. Rajaï F., Jean M., Moreau J.J., Roux S.. Force distribution in dense two-dimensional granular systems. Physical Review Letters. 1996;77(2):274-277.

7. Rajaï F., Wolf D.E., Jean M., Moreau J.J.. Bimodal character of stress transmission in granular packings. Physical Review Letters. 1998;80(1):61-64.

8. Bardet J.P.. Numerical simulations of the incremental responses of idealized granular materials. International Journal of Plasticity. 1994;10(8):879-908.

9. Sibille L., Hadda N., Nicot N., Tordesillas A., Darve F.. Granular plasticity, a contribution from discrete mechanics. Journal of the Mechanics and Physics of Solids. 2015c;75:119-139.

10. Iwashita K., Oda M.. Micro-deformation mechanism of shear banding process based on modified distinct element method. Powder Technology. 2000;109:192-205.

11. Govender N., Wilke D.N., Kok S.. Blaze-DEMGPU: Modular high performance DEM framework for the GPU architecture. SoftwareX. 2016;5:62 - 66 .

12. Mcdowell G. R., Falagush O., Yu H.-S.. A particle refinement method for simulating DEM of cone penetration testing in granular materials. Géotechnique Letters. 2012;2(3):141-147.

13. Rousseau J., Frangin E., Marin P., Daudeville L.. Multidomain finite and discrete elements method for impact analysis of a concrete structure. Engineering Structures. 2009;31(11):2735 - 2743.

14. ARROYO M., BUTLANSKA J., GENS A., CALVETTI F., JAMIOLKOWSKI M.. Cone penetration tests in a virtual calibration chamber. Géotechnique. 2011;61(6):525-531.

15. B. Chareyre, P. Villard. Dynamic Spar Elements and Discrete Element Methods in Two Dimensions for the Modeling of Soil-Inclusion Problems. Journal of Engineering Mechanics. 2005;131(7):689-698. 
16. Chevalier B., Villard P., Combe G.. Investigation of Load-Transfer Mechanisms in Geotechnical Earth Structures with Thin Fill Platforms Reinforced by Rigid Inclusions. International Journal of Geomechanics. 2011;11(3):239-250.

17. Constitutive equations for granular non-cohesive soils - Proceedings of the international workshop on constitutive equations for granular non-cohesive soils(22-24 July 1987, Cleveland)Balkema, Rotterdam1988.

18. Flavigny E., Desrues J., B. Palayer. Le sable d'Hostun RF. Revue Française de géotechnique. 1990;(53):67-70.

19. Lanier J., Zitouni Z.. Development of a data base using the Grenoble true triaxial apparatus. In: Saada A.S., Bianchini G.S, eds. Constitutive equations for granular non-cohesive soils - Proceedings of the international workshop on constitutive equations for granular non-cohesive soils, :47-58Balkema, Rotterdam; 1988; 22-24 July 1987, Cleveland.

20. Zitouni Z.. Comportement tridimensionnel des sables. PhD thesisUniversité Joseph Fourier - Grenoble 1Grenoble, France1988.

21. Darve F.. Geomaterials Constitutive Equations and Modellingch. Incrementally non-linear relationships, :213-238. Taylor and Francis books 1990.

22. Darve F., Flavigny E., Méghachou M.. Yield surfaces and principle of superposition: revisit through incrementally nonlinear constitutive relations. Int. J. Plasticity. 1995;11(8):927-948.

23. Darve F., Dendani H.. An incrementally non-linear constitutive relation and its predictions. In: Saada A.S., Bianchini G.S, eds. Constitutive equations for granular non-cohesive soils - Proceedings of the international workshop on constitutive equations for granular non-cohesive soils, :237-254Balkema, Rotterdam; 1988; 22-24 July 1987, Cleveland.

24. Cundall P.A., Strack O.D.L.. A discrete numerical model for granular assemblies. Géotechnique. 1979;29(1):47-65.

25. Górniak J., Villard P., Delmas P.. Coupled discrete and finite-element modelling of geosynthetic tubes filled with granular material. Geosynthetics International. 2016;23(5):362-380.

26. Villard P., Huckert A., Briançon L.. Load transfer mechanisms in geotextile-reinforced embankments overlying voids: Numerical approach and design. Geotextiles and Geomembranes. 2016;44(3):381-395.

27. Breugnot A., Lambert S., Villard P., Gotteland P.. A Discrete/continuous Coupled Approach for Modeling Impacts on Cellular Geostructures. Rock Mechanics and Rock Engineering. 2016;49(5):1831-1848.

28. Aboul Hosn R., Sibille L., Benahmed N., Chareyre B.. A discrete numerical model involving partial fluid-solid coupling to describe suffusion effects in soils. Computers and Geotechnics. 2018;95:30 - 39. 
29. Aboul Hosn R., Sibille L., Benahmed N., Chareyre B.. Discrete numerical modeling of loose soil with spherical particles and interparticle rolling friction.. Granular matter. 2017;19:4.

30. Combe G., Roux J.N.. Discrete-Element Modeling of Granular Materialsch. 6: Construction of Granular Assemblies under Static Loading. iSTE-Wiley 2011.

31. Chareyre B., Briançon L., Villard P.. Theoretical Versus Experimental Modeling of the Anchorage Capacity of Geotextiles in Trenches. Geosynthetics International. 2002;9(2):97-123.

32. Tong, A.-T. , Catalano, E. , Chareyre, B. . Pore-Scale Flow Simulations: Model Predictions Compared with Experiments on Bi-Dispersed Granular Assemblies. Oil Gas Sci. Technol. - Rev. IFP Energies nouvelles. 2012;67(5):743-752.

33. Salot C., Gotteland P., Villard P.. Influence of relative density on granular materials behavior: DEM simulations of triaxial tests. Granular Matter. 2009;11(4):221-236.

34. Bousquet H., Djeddid M., Khazar E., et al. Compilation des essais triaxiaux de révolution sur le sable d'Hostun RF. : Institut de mécanique de Grenoble; 1994.

35. Bianchini G., Puccini P., Saada A.. Test results. In: Saada A.S., Bianchini G.S, eds. Constitutive equations for granular non-cohesive soils - Proceedings of the international workshop on constitutive equations for granular non-cohesive soils, :89-97Balkema, Rotterdam; 1988; 22-24 July 1987, Cleveland.

36. Saleeb A.F., Lou K.A.. A simplified bounding surface plasticity model for predictions of sand behavior. In: Saada A.S., Bianchini G.S, eds. Constitutive equations for granular non-cohesive soils - Proceedings of the international workshop on constitutive equations for granular non-cohesive soils, :593-613Balkema, Rotterdam; 1988; 22-24 July 1987, Cleveland.

37. Cambou B., Jafari K.. A constitutive model for granular materials based on two plasticity mechanisms. In: Saada A.S., Bianchini G.S, eds. Constitutive equations for granular non-cohesive soils - Proceedings of the international workshop on constitutive equations for granular non-cohesive soils, :149-167Balkema, Rotterdam; 1988; 22-24 July 1987, Cleveland.

38. Roux J.N., G. Combe. How granular materials deform in quasistatic conditions. In: J. D. Goddard P. Giovine, ed. IUTAMISIMM Symposium on Mathematical Modeling and Physical Instances of Granular Flow, :260AIP Conference Proceedings (vol. 1227); 2010.

39. Benahmed N., Nguyen T.K., Hicher P.Y., Nicolas M.. An experimental investigation into the effects of low plastic fines content on the behaviour of sand/silt mixtures. European Journal of Environmental and Civil Engineering. 2015;19(1):109128. 
40. Aris M., Benahmed N., Bonelli N.. Experimental geomechanics: a laboratory study on the behaviour of granular material using bender elements. European Journal of Environmental and Civil Engineering. 2012;16(1):97-110.

41. Benahmed N., Canou J., Dupla J.C.. Structure initiale et propriétés de liquéfaction statique d'un sable. Comptes Rendus Mécanique. 2004;332(11):887 - 894. 